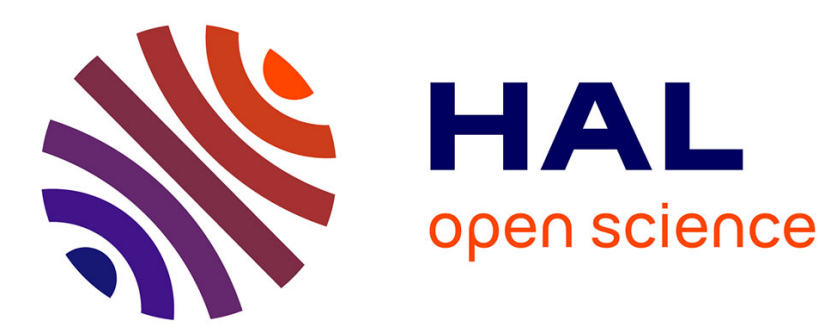

\title{
Integrated vehicle dynamics control via coordination of active front steering and rear braking
}

Moustapha Doumiati, Olivier Sename, Luc Dugard, John Jairo Martinez

Molina, Peter Gaspar, Zoltan Szabo

\section{- To cite this version:}

Moustapha Doumiati, Olivier Sename, Luc Dugard, John Jairo Martinez Molina, Peter Gaspar, et al. Integrated vehicle dynamics control via coordination of active front steering and rear braking. European Journal of Control, 2013, 19 (2), pp.121-143. 10.1016/j.ejcon.2013.03.004 · hal-00759487

\section{HAL Id: hal-00759487 \\ https://hal.science/hal-00759487}

Submitted on 3 Dec 2012

HAL is a multi-disciplinary open access archive for the deposit and dissemination of scientific research documents, whether they are published or not. The documents may come from teaching and research institutions in France or abroad, or from public or private research centers.
L'archive ouverte pluridisciplinaire HAL, est destinée au dépôt et à la diffusion de documents scientifiques de niveau recherche, publiés ou non, émanant des établissements d'enseignement et de recherche français ou étrangers, des laboratoires publics ou privés. 


\title{
Integrated vehicle dynamics control via coordination of active front steering and rear braking
}

\author{
Moustapha Doumiati, Olivier Sename, Luc Dugard, John Martinez, ${ }^{a}{ }^{*}$ \\ Peter Gaspar, Zoltan Szabo ${ }^{b}$ \\ ${ }^{a}$ Gipsa-Lab UMR CNRS 5216, Control Systems Department, \\ 961 Rue de la Houille Blanche, 38402 Saint Martin d'Hères, France, \\ Email: moustapha.doumiati@gmail.com, \{surname.name\}@gipsa-lab.grenoble-inp.fr \\ ${ }^{b}$ Computer and Automation Research Institue, Hungarian Academy of Sciences, \\ Kende u. 13-17, H-1111, Budapest, Hungary, \\ Email: gaspar, szabo@sztaki.hu
}

January 26, 2012

\begin{abstract}
This paper investigates the coordination of active front steering and rear braking in a driverassist system for vehicle yaw control. The proposed control system aims at stabilizing the vehicle while achieving a desired yaw rate. During normal driving situations, active steering control is involved for steerability enhancement. However, when the vehicle reaches the handling limits, both steering and braking collaborate together to ensure vehicle stability. The coordination of these actuators is achieved through a suitable gain scheduled $L P V$ (Linear Parameter Varying) controller. The controller is synthesized within the LMI (Linear Matrix Inequalities) framework, while warranting robust $\mathscr{H}_{\infty}$ performances. Time and frequency simulation results show the effectiveness of the proposed control scheme when the vehicle is subject to different critical driving situations.
\end{abstract}

Keywords: Integrated control, Vehicle dynamics, LPV system, $\mathscr{H}_{\infty}, L M I$

${ }^{*}$ M. Doumiati and O. Sename are corresponding authors 


\section{Introduction}

\subsection{Motivation}

A trend in modern vehicles is the application of active safety systems to improve vehicle handling, stability and comfort. Nowadays, many advanced active chassis control systems have been developed and brought into the market: i.e, ABS (Anti-lock Braking System) prevents wheel lock-up, and ESC (Electronic Stability Control) enhances vehicle lateral stability. The development of chassis control systems is still an object of intense research activities from both industrial and academic sides. The various vehicle dynamics control systems can be classified into three areas: longitudinal, lateral and vertical control in terms of the three translational vehicle motions. This work focusses on active control of vehicle handling and lateral vehicle dynamics.

Safety of ground vehicles requires the improvement of yaw stability by active control. The basic idea is to assist the vehicle handling to be close to linear vehicle characteristics that are familiar to the driver (referred to as a nominal vehicle behavior), and to restrain the vehicle lateral dynamics to be within a stable handling region in aggressive maneuvers. Several actuators, such as active suspension, active steering and active braking could be used for yaw rate control. An active suspension system, by controlling the wheel load, may improve the lateral dynamics of the vehicle [34]. An Active Steering $(A S)$ system, by controlling the steering angles of the wheels, has great influence on the lateral behavior of the vehicle. Finally, an active braking system like the Direct Yaw Control (DYC), by using differential braking, is very effective for lateral stability of the vehicle.

\subsection{Toward integrated control and related works}

Based on the above discussion, this study focuses on two main methods to control the yaw moment in order to improve vehicle handling and stability.

The first one is the DYC technique that utilizes differential braking forces between the left and the right sides of the vehicle to produce the required corrective yaw moment. DYC exploits the interaction between longitudinal and lateral tire forces to influence the vehicle handling. On this topic, some relevant results can be found in the literature, i.e, Predictive control [1], Fuzzy control, Sliding mode control [7], Internal Model Control [6] and $\mathscr{H}_{\infty}$ control [30] and LPV [8, 9] were investigated.

The second method is the $A S$ that regulates the tire slip angle and affects the vehicle handling be- 
havior by directly modulating the generation of lateral tire forces. Three active steering schemes exist: Active Front Steering $(A F S)$ [21, 16, 10], Active Rear Steering (ARS) [24] and Active Four Wheel Steering $(W 4 S)$ [25]. This study mainly focusses on the most commonly used $A F S$ approach. This latter may be formulated using disturbance observer control method [21, 22], sliding mode control [23], predictive control [16], or other control techniques. Such active handling control usually serves a steering support system by applying an additional steering angle to the driver's steer command. Due to the extra steering action, the potential of $A F S$ will be easily usable once Steer-by-wire technology is established. In [39], the authors discuss in details the capability of steering and braking smart actuators to control the vehicle yaw motion.

During high lateral acceleration, due to the inherent nonlinear characteristics and tire saturations, $A S$ is no longer able to produce enough lateral force by steering to hold on the vehicle in a turn. In other words, $A S$ cannot keep the vehicle under control when the handling limit is reached, and consequently $A S$ performance is limited within the linear vehicle handling region (low to mid-rang lateral accelerations). On the other hand, $D Y C$ is shown to be effective in both vehicle linear/nonlinear regions, however, it is only desirable for limit handling rather than for normal driving situations. This is due to the braking effect that wears out the tire and interferes with the longitudinal vehicle dynamics. Moreover, $D Y C$ causes the vehicle to slow down significantly, and this may be objectionable and not desirable for the driver. Consequently, $A S$ and $D Y C$ control techniques are optimized individually in specific handling regions, and the maximum benefit could be gained through the coordinated/integrated use of both methods of corrective yaw motion generation in the control strategy. Practically, nowadays integrated control may be possible due to the improved capabilities of the vehicle Electronic Control Units capabilities that permit to implement control algorithms that coordinate/integrate multiple actuators. Moreover a comparative study of $A F S$ and $A R S$ when coordinated with $D Y C$, came to a conclusion that $A F S$ is more suitable to be coordinated with $D Y C$ than $A R S$ in terms of assisting DYC in maintaining vehicle stability [40]. In the present study, to avoid interferences between $A F S$ and $D Y C$, rear braking is only used. Consequently, the integrated control of front steering and rear braking actions is a relevant choice for vehicle stability.

Research in integrated vehicle dynamics control and global chassis control is a very active field in the recent years. Concerning the lateral behavior, different actuators configuration have been considered using front/rear steering and braking torque distribution (front/rear, differential braking) [22, 20, 11, $39,43,44,33$ ] but also more recently with differential tire slip [26] if electrical in-wheel-motors are considered. Advanced control methods have then been developed to solve this complex control problem 
for a MIMO system, such as optimal control [44], control allocation [43, 39], Model Predictive Control [11], and robust control $[22,20]$. Some of the previous existing studies, such as [23, 20], develop separately both $D Y C$ and $A F S$ systems, and then propose a switching strategy between both stand-alone systems, according to the driving situations. However, for this strategy, the internal system stability may be in question due to the switching process. Other publications, such [44], propose control strategies that demand online calculations, which may cause implementation difficulties. In that regard, this paper builds a controller that does not involve any online optimization process, and thus is suitable for realtime applications. The developed controller also takes into account the passengers comfort, in contrast to many papers such as $[11,44,45]$ that does not consider this issue.

\subsection{Contributions}

The present work deals with the design of a new vehicle chassis control scheme that integrates and coordinates rear braking and front steering. The control scheme is built on a MIMO (Multi Input Multi Output) gain scheduled controller worked out on the basis of a 2-DOF (Degree-Of-Freedom) linear planar vehicle model. The control goal is to restore the yaw rate of the vehicle as close as possible to the nominal motion expected by the driver, and to limit the use of the braking actuator only when the vehicle goes toward instability. Judging the vehicle stability region is deduced from the phase-plane of the sideslip angle and its time derivative, which is used to monitor the car dynamical behavior.

The proposed controller is then formulated as a unified Linear Parameter Varying $(L P V)$ controller structure in order to coordinate steering and braking actuators. The single exogenous scheduling control parameter feeding the $L P V$ controller is a "monitor" function of the sideslip angle dynamics. The controller is synthesized within the $\mathscr{H}_{\infty}$ framework for polytopic systems, and then ensures the internal closed-loop stability of the MIMO system. It also emphasizes some inherent robustness properties w.r.t parameter variations, and will simplify the engineer design and reduce the development time in making actuators cooperate.

The present work is an extension of the preliminary results obtained by the same authors in previous papers $[32,33,13]$. The brought contributions include a better interpretation of the lateral vehicle dynamics behavior and of the braking torque distribution, as well as the development of a new LPV strategy of steering/braking actuators collaboration. Indeed in [32] the braking system acts first when dangerous situation is detected, and if it is not efficient enough to stabilize the vehicle, then, by monitoring the braking torque efficiency, the steering system is activated to handle the dynamical problem . 


\begin{tabular}{llcl}
\hline Symbol & Value & Unit & Signification \\
\hline \hline$m$ & 1535 & $\mathrm{~kg}$ & vehicle mass \\
$m_{r}$ & 648 & $\mathrm{~kg}$ & vehicle rear mass \\
$I_{z}$ & 2149 & $\mathrm{~kg} \cdot \mathrm{m}^{2}$ & vehicle yaw inertia \\
$C_{f}$ & 40000 & $\mathbf{N} / \mathbf{r a d}$ & cornering stiffness of front tires \\
$C_{r}$ & 40000 & $\mathbf{N} / \mathbf{r a d}$ & cornering stiffness of rear tires \\
$l_{f}$ & 1.4 & $m$ & distance COG - front axle \\
$l_{r}$ & 1 & $m$ & distance COG - rear axle \\
$t_{r}$ & 1.4 & $m$ & rear axle length \\
$h$ & 0.5 & $m$ & height of the center of gravity \\
\hline$\mu$ & {$[2 / 5 ; 1]$} & - & tire/road contact friction interval \\
$v$ & {$[50 ; 130]$} & $\mathrm{km} / \mathrm{h}$ & vehicle velocity interval \\
$g$ & 9.81 & $\mathrm{~m} / \mathrm{s}^{2}$ & gravitational acceleration \\
\hline
\end{tabular}

Table 1: Notations and vehicle parameters.

In $[13,33]$, the LPV approach is used to coordinate 3 control inputs, i.e. the rear-(left and right) braking torques and the additive steering angle, through 2 high level parameters. Moreover the steering action is considered only in case of emergency situations, and the (left or right) braking torque is activated according to the value of the yaw rate error. Here 2 control inputs are considered (the steering angle and the yaw moment), and only a single parameter is used (in order to weight the braking action), which means that the steering angle will always be used, while the yaw moment will be used only in case of dangerous situations. The application of the (left or right) braking torques is decided at a lower level.

The behavior of the vehicle with the proposed control scheme has been evaluated subject to various driving situations, using simulations through time-domain and frequency-domain analysis on a full nonlinear vehicle model presented in [33]. The obtained results confirm the effectiveness of the proposed integrated control.

The paper is structured as follows. Section II first introduces the global control scheme, and then a global LPV controller is developed. Performance analysis is done in Section III through different simulations. Conclusions and discussions are given in Section IV.

\section{Paper notations:}

Throughout the paper, the following notations will be adopted: index $i \in\{f, r\}$ and $j \in\{l, r\}$ are used to identify vehicle front, rear and left, right positions respectively. Table 1 summarizes the vehicle parameters, and the notations adopted in the paper. 


\section{Control system design}

The control system is shown in the block diagram of figure 1. This architecture includes a controller and an estimator. Signals such as steering wheel angle, wheel speeds, yaw rate, longitudinal and lateral accelerations are available at reasonable costs or already exist on vehicles equipped with an ESC system. Let mention that the sideslip angle is a difficult and an expensive measurement to achieve in practice, and thus, it must be observed using a virtual sensor. The observer design is not part of the paper contribution and could be found in different papers as (see $[31,27,19,29,14])$

The main goal of the proposed control system is to make the actual yaw rate, $\dot{\psi}$, to follow the desired yaw rate, $\dot{\psi}_{d}$. In other words, the controller must track the reference yaw rate intended by the driver through driving the tracking error between the actual and desired yaw rate to zero. The chosen yaw rate reference model is adopted to keep the vehicle within the linear region that is familiar to the driver. Another purpose of the controller is to limit the vehicle sideslip angle, $\beta$, to be within an acceptable region to prevent vehicle spin.

As seen in figure 1, the controller responds to the yaw rate error, and its outputs are the active steer angle and the braking moment. In the following, each block of the control scheme is described in details.

\subsection{Reference model}

To obtain a desired vehicle behavior, it is necessary that the yaw rate follows its target value, $\dot{\psi}_{d}$. Usually, the driver attempts to control the yaw rate of its vehicle during normal and moderate cornering from the steerability point of view. Therefore, the reference model must reflect the desired relationship between the driver steer inputs and the vehicle yaw rate, while keeping the vehicle in a safe operating region. The 2-DOF (Degree Of Freedom) classical linear bicycle model [15], depicted in figure 2, owns this feature and is thus adopted to generate the yaw rate target value. More comments on using the bicycle model to generate the reference yaw-rate with respect to the effects of unmodeling and modeling errors effects can be found in [6].

The equations governing the lateral and yaw motions in a bicycle model can be expressed as:

- Equation of lateral motion:

$$
m v(\dot{\beta}-\dot{\psi})=F y_{f}+F y_{r}
$$

- Equation of yaw motion:

$$
I_{z} \ddot{\psi}=l_{f} F y_{f}-l_{r} F y_{r}
$$




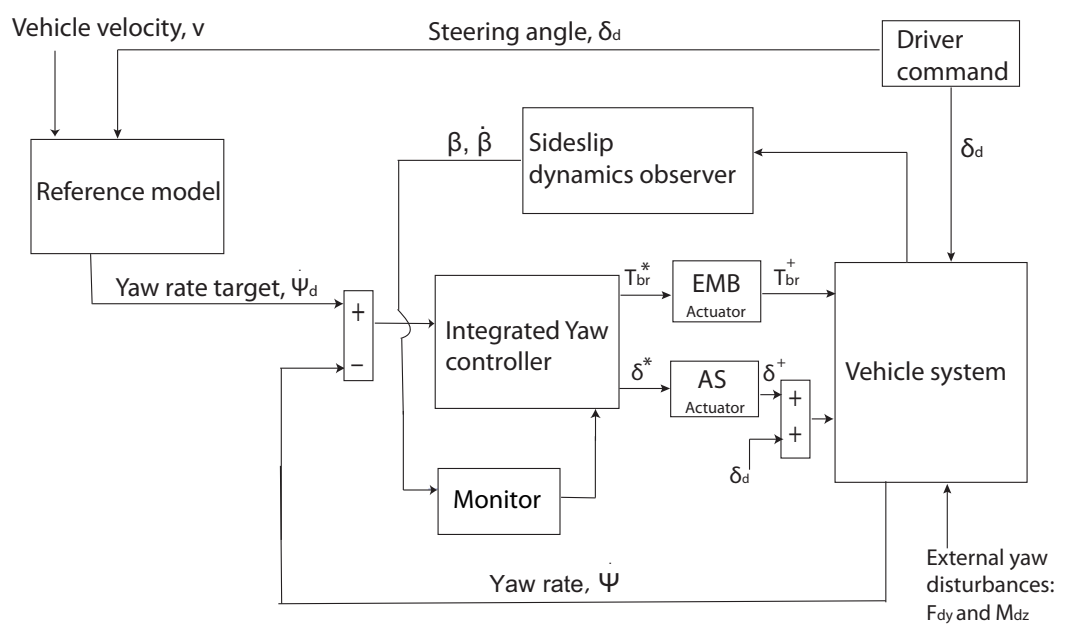

Figure 1: Global control scheme.

where $\dot{\psi}$ is the yaw rate, $\beta$ the vehicle side slip angle, $v$ the longitudinal vehicle speed, $m$ the vehicle total mass, $F y_{f}$ and $F y_{r}$ are the front and rear lateral tire forces.

\section{Linear model assumptions:}

- Assuming a linear tire model, $F y_{f}$ and $F y_{r}$, are given by:

$$
F y_{f, r}=C_{f, r} \alpha_{f, r}
$$

where $\alpha_{f}$ and $\alpha_{r}$ are the front and rear sideslip angles.

- Assuming a small body sideslip angle $\beta$ leads to:

$$
\left\{\begin{array}{l}
\alpha_{f}=\delta_{d}-\beta-l_{f} \frac{\dot{\psi}}{v_{x}} \\
\alpha_{r}=-\beta+l_{r} \frac{\dot{\psi}}{v_{x}}
\end{array}\right.
$$

In this reference model, $\dot{\psi}_{d}$ is function of the driver steering wheel angle $\delta_{d}$, and of the vehicle forward speed $v_{x}$.

Since the lateral acceleration of the vehicle cannot exceed the maximum friction coefficient $\mu$, the desired yaw rate must be limited by the following value [35]:

$$
\left|\dot{\psi}_{d, \max }\right| \leq\left|\mu g / v_{x}\right|
$$




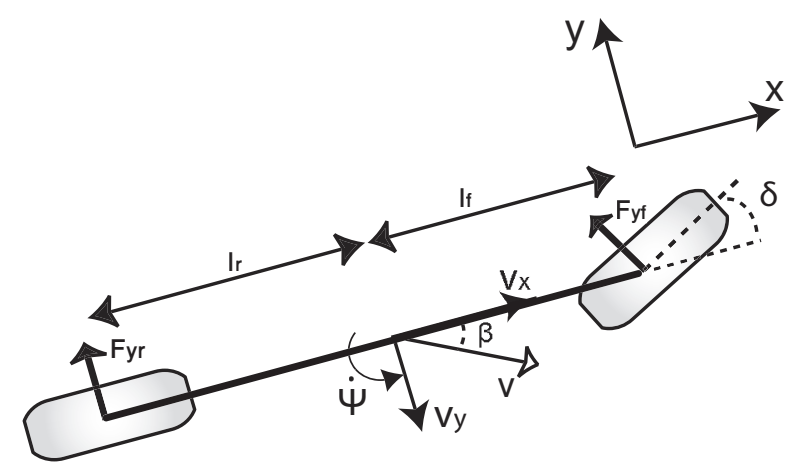

Figure 2: 2-DOF model of lateral vehicle dynamics.

Consequently, the desired $\dot{\psi}_{d}$ constrained within upper and lower bounds, constitutes the reference signal to be tracked by the yaw stability controller.

\subsection{Yaw controller}

The yaw controller is designed so that the vehicle follows the reference yaw rate by driving the tracking error between the actual and desired yaw rate to zero. Figure 3 shows the whole hierarchical structure of the controller, designed in 2 layers:

a) The upper-level controller defines the amount of the active steer angle $\delta^{*}$, and the corrective yaw moment $M_{z}^{*}$, needed to achieve a good tracking of the yaw-rate set-point.

Note that when the vehicle is within the linear region, the controller ensures steerability and only steering is used to follow the desired response. However, when the vehicle reaches the handling limits, steering and braking act together to maintain the vehicle stability.

b) The lower-level controller converts the stabilizing yaw moment generated by the upper-level controller into an effective braking torque, and it decides which wheel must be braked to counteract the undesired yaw motion.

Remark 1 Notice that the main contribution relies in the synthesis of the LPV upper-level controller which ensures the actuator coordination through the use of a parameter $\rho$ depending on a stability index of the vehicle. The lower-level controller chosen here is a simple one to distribute efficiently the braking torque at a single wheel. However, since the yaw moment is a control input, this lower-lever controller could be replaced by more advanced strategies including control allocation [43], or some other structure such as differential braking could be considered as proposed for instance in [8, 9, 6, 7]. 


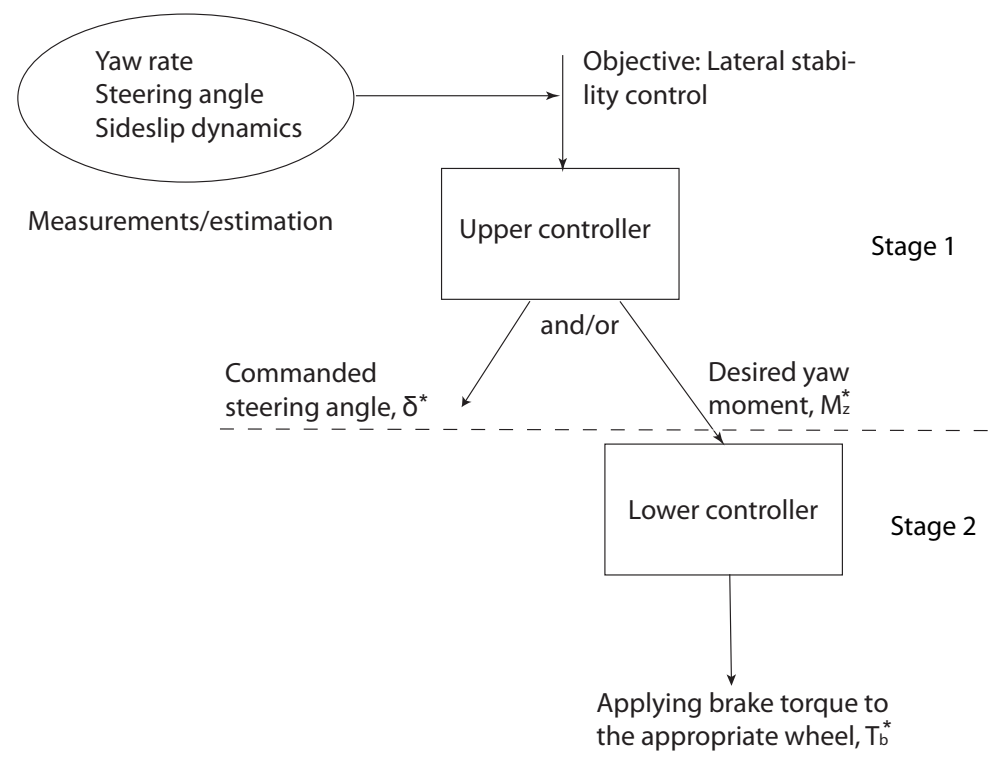

Figure 3: Yaw controller architecture.

\subsection{1 upper-level controller: $L P V / \mathscr{H}_{\infty}$ controller design}

The first step in a control design consists in defining of the control objectives. The proposed integrated control system is designed to achieve the following goals:

- Improvement of the vehicle handling and stability by:

- making the yaw rate tracking the desired value (given in terms of the response of a bicycle model with linear tire properties);

- making the sideslip angle small for stability.

- Coordination of Steering/braking control in order to minimize the influence of brake intervention on the longitudinal vehicle dynamics (passengers comfort).

- Activation of steering control in a frequency band where the driver cannot act (driver comfort as in [33]).

- Rejection of yaw moment disturbances.

The 2-DOF linear bicycle model described in Section 2 is used for the control synthesis. Although the bicycle model is relatively simple, it captures the important features of the lateral vehicle dynamics. Taking into account the controller structure and objectives, this model is extended to include:

- the direct yaw moment input $M_{z}^{*}$, 


$$
\left[\begin{array}{c}
\ddot{\psi} \\
\dot{\beta}
\end{array}\right]=\left[\begin{array}{cc}
-\frac{l_{f}^{2} C_{f}+l_{r}^{2} C_{r}}{I_{z} v} & \frac{l_{r} C_{r}-l_{f} C_{f}}{I_{z}} \\
1+\frac{l_{r} C_{r}-l_{f} C_{f}}{m v^{2}} & -\frac{C_{f}+C_{r}}{m v}
\end{array}\right]\left[\begin{array}{c}
\dot{\psi} \\
\beta
\end{array}\right]+\left[\begin{array}{c}
\frac{l_{f} C_{f}}{I_{z}} \\
\frac{C_{f}}{m v}
\end{array}\right] \delta^{*}+\left[\begin{array}{c}
\frac{1}{I_{z}} \\
0
\end{array}\right] M_{z}^{*}+\left[\begin{array}{c}
\frac{1}{I_{z}} \\
\frac{1}{m v}
\end{array}\right]\left[\begin{array}{c}
M_{d z} \\
F_{d y}
\end{array}\right]
$$

- a lateral disturbance force $F_{d y}$ and a disturbance moment $M_{d z} . F_{d y}$ affects directly the sideslip motion, while $M_{d z}$ influences directly the yaw motion.

In the following, the extended linear bicycle model given in system (6) is used for controller synthesis. To synthesize the upper control, the $\mathscr{H}_{\infty}$ control performance is used to provide robustness to model uncertainty and external disturbances. For more information about the robust $\mathscr{H}_{\infty}$ theory, reader can refer to $[41,2]$. In the following, the generalized plant together with the performance weighting functions, called here $\sum_{g}$, is presented and illustrated in figure 4. Dynamics of the actuators are neglected during the controller design process.

In order to formulate the standard structure for the $\mathscr{H}_{\infty}$ controller defined in figure 4 , the weighting functions $W_{1}, W_{2}, W_{3}$, and $W_{4}$ are defined to characterize the performance objectives and the actuator limitations (the actuator descriptions are given in Subsection 2.5):

- $W_{1}$ weights the sideslip angle signal, $\beta$ :

$$
W_{1}(s)=2 \forall s
$$

It restricts the body sideslip angle and the vehicle lateral velocity evolution. This angle is penalized during the controller setup since not only the turning rate response is important during cornering, but it is also desired to have low sideslip angle.

- $W_{2}$ weights the yaw rate error signal, $e_{\psi}\left(e_{\psi}=\dot{\psi}_{d}-\dot{\psi}\right)$ :

$$
W_{2}(s)=\frac{s / M+w_{0}}{s+w_{0} A}
$$

where $M=2$ for a good robustness margin, $A=0.1$ so that the tracking error is less than $10 \%$, and the required bandwidth $w_{0}=70 \mathrm{rad} / \mathrm{s} . W_{2}$ is shaped in order to reduce the yaw rate error.

- $W_{3}$ weights the braking control signal, $M_{z}^{*}$, according to a scheduling parameter $\rho$ :

$$
W_{3}(s, \rho)=\rho \frac{s /\left(2 \pi f_{2}\right)+1}{s /\left(\alpha 2 \pi f_{2}\right)+1}
$$

where $f_{2}=10 \mathrm{~Hz}$ is the braking actuator cut-off frequency and $\alpha=100$. These parameters are 


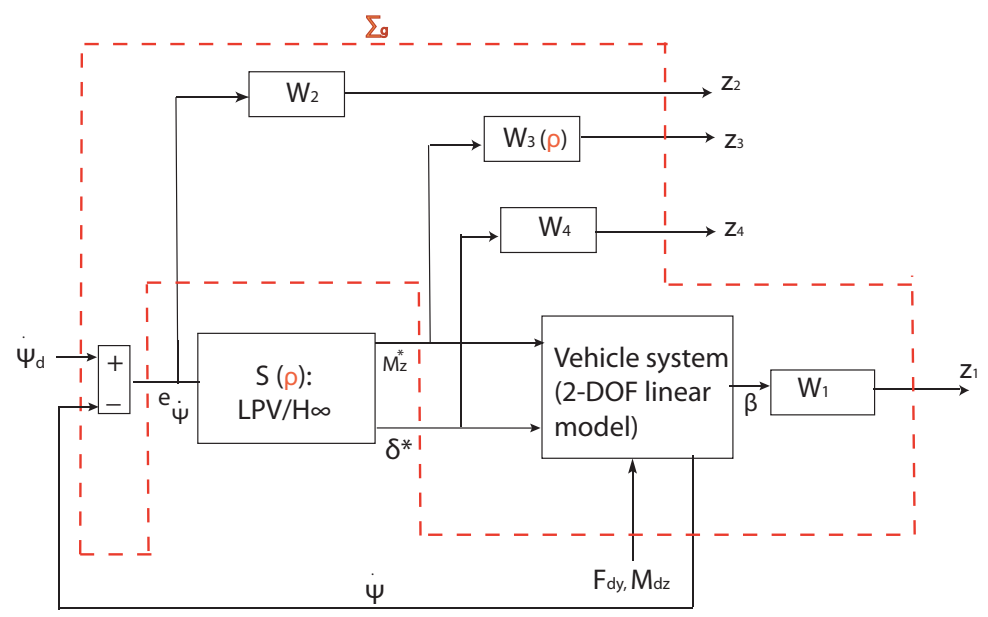

Figure 4: Generalized plant model for synthesis.

chosen to handle braking actuator dynamic limitations (see Subsection 2.5).

$W_{3}$ is linearly parameterized by the considered varying parameter $\rho($.$) , where \rho \in\{\underline{\rho} \leq \rho \leq \bar{\rho}\}$ (with $\underline{\rho}=10^{-5}$ and $\bar{\rho}=10^{-3}$ ). Then, when $\rho=\bar{\rho}$, the braking input is penalized, on the contrary, when $\rho=\underline{\rho}$, the braking control signal is relaxed.

- $W_{4}$ weights the steering control signal, $\delta^{*}$ :

$$
\begin{aligned}
W_{4}(s) & =G_{\delta}^{0} \frac{\left(s / 2 \pi f_{3}+1\right)\left(s / 2 \pi f_{4}+1\right)}{\left(s / \alpha 2 \pi f_{4}+1\right)^{2}} \\
G_{\delta}^{0} & =\frac{\left(\Delta_{f} / \alpha 2 \pi f_{4}+1\right)^{2}}{\left(\Delta_{f} / 2 \pi f_{3}+1\right)\left(\Delta_{f} / 2 \pi f_{4}+1\right)} \\
\Delta_{f} & =2 \pi\left(f_{4}+f_{3}\right) / 2
\end{aligned}
$$

where $f_{3}=1 \mathrm{~Hz}$ is the lower limit of the actuator intervention, and $f_{4}=10 \mathrm{~Hz}$ is the steering actuator bandwidth (see Subsection 2.5). This filter is chosen to allow the steering control to act only in the $\left[f_{3}, f_{4}\right]$ frequency range, where the driver cannot act. Thus, it is ensured that the steering action is comfortable for the driver. This filter design is inspired from [21, 22].

Since the generalized plant $\sum_{g}$ is $L P V$ [3], it can be formulated as:

$$
\Sigma_{g}(\rho):\left[\begin{array}{c}
\dot{x} \\
z \\
y
\end{array}\right]=\left[\begin{array}{ccc}
A & B_{1} & B_{2}(\rho) \\
C_{1}(\rho) & D_{11} & D_{12}(\rho) \\
C_{2} & D_{21} & 0
\end{array}\right]\left[\begin{array}{l}
x \\
w \\
u
\end{array}\right]
$$

where $x$ includes the state variables of the system and of the weighting functions, $w=\left[\dot{\psi}_{d}, F_{d y}, M_{d z}\right]^{T}$ is the exogenous input vector, $u=\left[\delta^{*}, M_{z}^{*}\right]^{T}$ represents the control input signals, $y=e_{\psi}$ is the measure- 
ment vector, and $z=\left[z_{1}, z_{2}, z_{3}, z_{4}\right]^{T}$ contains the weighted controlled outputs which have to be as small as possible.

Note that the matrices $B_{2}, C_{1}$ and $D_{12}$ depend on $\rho$, which does not cope with the requirements of the $H_{\infty}$ synthesis for polytopic systems. However, as mentioned in [2], this asumption can be relaxed using some filter on the control input, which has been done here.

Problem resolution: $L M I$ based $L P V / \mathscr{H}_{\infty}$ :

The $\mathscr{H}_{\infty}$ problem consists in finding a stabilizing controller, $S(\rho)$ (see figure 4), scheduled by the parameter $\rho$ :

$$
S(\rho):\left[\begin{array}{c}
\dot{x}_{c} \\
u
\end{array}\right]=\left[\begin{array}{cc}
A_{c}(\rho) & B_{c}(\rho) \\
C_{c}(\rho) & 0
\end{array}\right]\left[\begin{array}{l}
x_{c} \\
y
\end{array}\right]
$$

that minimizes the $\mathscr{H}_{\infty}$ norm of the closed-loop $L P V$ system formed by the interconnection of equations (11) and (12), where $u=\left[\delta^{*}, M_{z}^{*}\right]^{T}$ and $y=e_{\psi}$.

Finding such a controller can be done by applying the well known Bounded Real Lemma (BRL) extended to LPV systems. According to system (11), and via the change of basis expressed in [38] , a non conservative $L M I$ that expresses the same problem as the BRL can be formulated in (15) and solved by a Semi-Definite Program (SDP), while minimizing $\gamma$ for $\rho \in \Omega=\{\underline{\rho}, \bar{\rho}\}$.

The polytopic approach to this problem consists in finding $\widetilde{\mathbf{A}}, \widetilde{\mathbf{B}}$ and $\widetilde{\mathbf{C}}$ at each vertex of the polytope described by $\rho \in\{\underline{\rho}, \bar{\rho}\}$, by using a common Lyapunov function, i.e common $X>0$ and $Y>0$. Then, the solution can be obtained by solving the system (13) at each vertex $(\underline{\rho}, \bar{\rho})$ of the convex hull $\Omega$ :

$$
\left\{\begin{aligned}
C_{c}(\rho) & =\widetilde{C}(\rho) M^{-T} \\
B_{c}(\rho) & =N^{-1} \widetilde{B}(\rho) \\
A_{c}(\rho) & =N^{-1}\left(\widetilde{A}(\rho)-Y A X-N B_{c}(\rho) C_{2} X\right. \\
& \left.-Y B_{2} C_{c}(\rho) M^{-T}\right) M^{-T}
\end{aligned}\right.
$$

where $M$ and $N$ are defined by the user so that $M N^{T}=I-X Y$. See [38] for more details on the computation solution. According to the polytopic approach, the applied controller, $S(\rho)$, is a convex combination of the controllers synthesized at the vertices $\{\underline{\rho}, \bar{\rho}\}[3]$ :

$$
S(\rho)=\frac{|\rho-\bar{\rho}|}{(\bar{\rho}-\underline{\rho})} S(\underline{\rho})+\frac{|\rho-\underline{\rho}|}{(\bar{\rho}-\underline{\rho})} S(\bar{\rho})
$$

where $S(\underline{\rho})$ and $S(\bar{\rho})$ are the solutions of the polytopic problem evaluated at the vertices. 


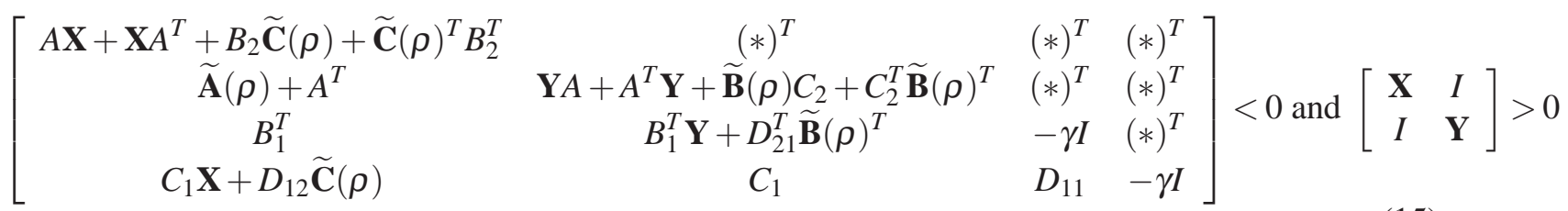
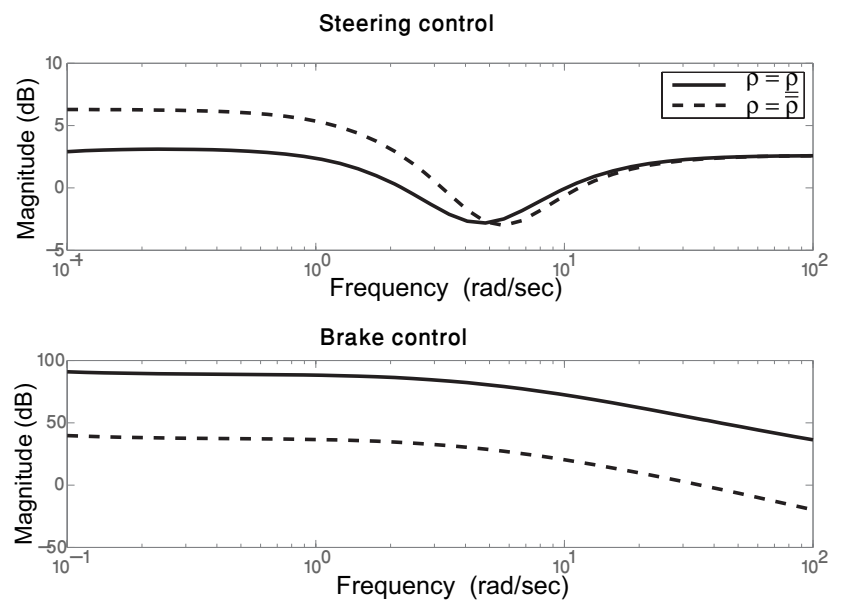

Figure 5: Bode diagrams of the controller outputs $\delta^{*}$ and $M_{z}^{*}$.

By solving offline the $L M I$ given in (15) for the LPV system (11) using Yalmip interface [28] and SeDumi solver [42], one obtains the optimal value $\gamma_{o p t}=2.4$. The Bode diagram given in figure 5 shows the steering and braking controller outputs w.r.t $\rho$. As the braking weight is parameter dependent, it is shown that when $\rho=\bar{\rho}$ the braking signal is attenuated, and conversely, when $\rho=\rho$ the braking gain is larger. As a consequence, when $\rho$ is low (resp. high), the braking is activated (resp. disabled). Intermediate values will give intermediate behaviors. Remember that, for any $\rho \in\{\underline{\rho} \leq \rho \leq \bar{\rho}\}$, the closed loop stability is ensured, thanks to the $L P V$ design and the polytopic approach.

According to the sensitivity functions Bode diagrams given in figure 6, it is interesting to make the following deductions:

- The sideslip angle, $\beta$, and the yaw rate error signal, $e_{\psi}$, are well attenuated (see figures 6 (a) and 6(b), respectively).

- $\left|e_{\psi} / \dot{\psi}_{d}\right|$ emphasizes that the yaw rate tracking performance satisfies the required specification (see figure 6(a)).

- The braking control is activated for $\rho=\underline{\rho}$, and it is limited for $\rho=\bar{\rho}$ (see figure 6(c)). Note that intermediate values of $\rho \in[\underline{\rho}, \bar{\rho}]$ give intermediate behaviors. 
- The steering control is activated especially in the specified frequency range $[1 \mathrm{~Hz}, 10 \mathrm{~Hz}]$ where the driver cannot act (see figure 6(d)).

\subsection{2 lower-level controller: braking control scheme}

The desired yaw moment command, $M_{z}^{*}$, produced by the upper-level controller can be generated by applying a torque difference between the two sides of the vehicle. Let us first convert $M_{z}^{*}$ to a torque and then select the appropriate wheels to be braked.

For simplicity, the quasi-static rotational dynamics of the wheel, at position $\{i, j\}$, is employed and given as:

$$
T_{b, i j}=R_{w} F x_{i j}
$$

where $R_{w}$ is the effective tire radius and $F x_{i, j}$, the longitudinal tire force.

Assuming a symmetric vehicle mass distribution, the corrective yaw moment demanded by the controller can be expressed as:

$$
M_{z}^{*}=\frac{t \Delta F_{x}}{2}
$$

where $t$ is the vehicle's rear axle length, $\Delta F_{x}$ is the longitudinal force between the left and right driving wheels of the same axle. Thus, the corresponding torque difference, between the left and right sides, can be expressed in terms of $M_{z}^{*}$, and takes the form:

$$
\Delta T=T_{\text {left }}-T_{\text {right }}=\frac{2 M_{z}^{*} R_{w}}{t} .
$$

In the following, the control law will be designed in order to select the most effective wheels to apply the brake torque, according to both following situations:

- Understeer condition: in this case, the absolute value of the vehicle yaw rate, $\dot{\psi}$, is always smaller than the absolute value of the desired vehicle yaw rate, $\dot{\psi}_{d}$. Therefore, the inner wheels will be chosen to generate a pro-cornering yaw moment.

- Oversteer condition: in this condition, the absolute value of the vehicle yaw rate, $\psi$, is always greater than the absolute value of desired vehicle yaw rate, $\dot{\psi}_{d}$. Hence, the outer wheels will be selected to generate a contra-cornering yaw moment.

In both of these two dynamic conditions, either both wheels or one wheel (on one side) can be braked to generate $M_{z}^{*}$. However, from an optimal control point of view, it is recommended to use one wheel to 

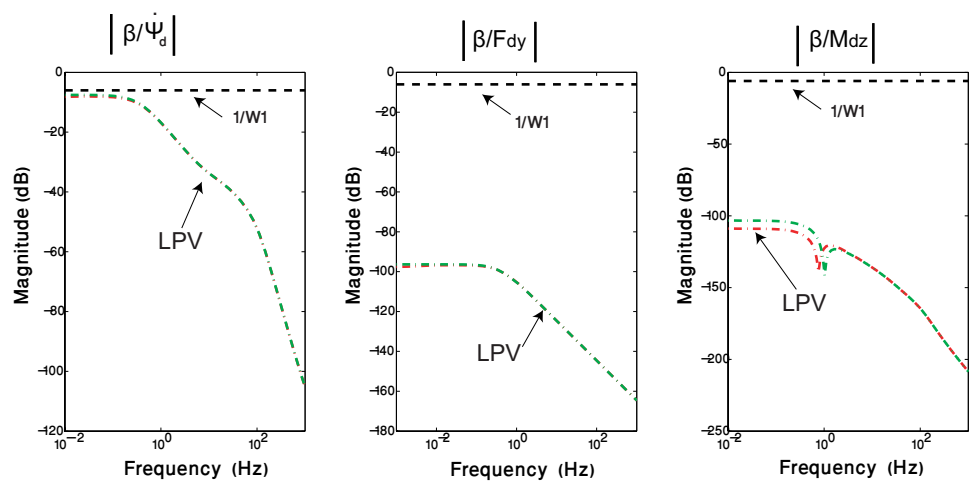

(a) Closed loop transfer functions between $\beta$ and exogenous inputs.
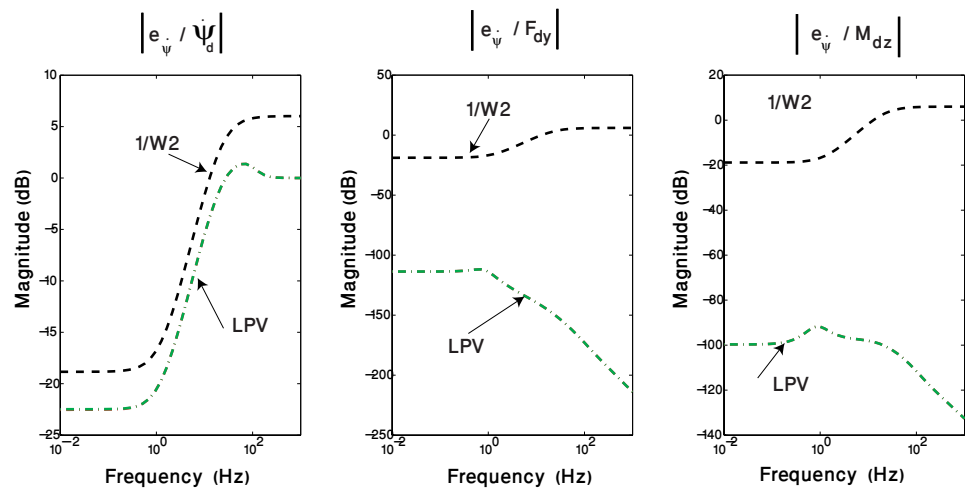

(b) Closed loop transfer functions between $e_{\dot{\psi}}$ and exogenous inputs.
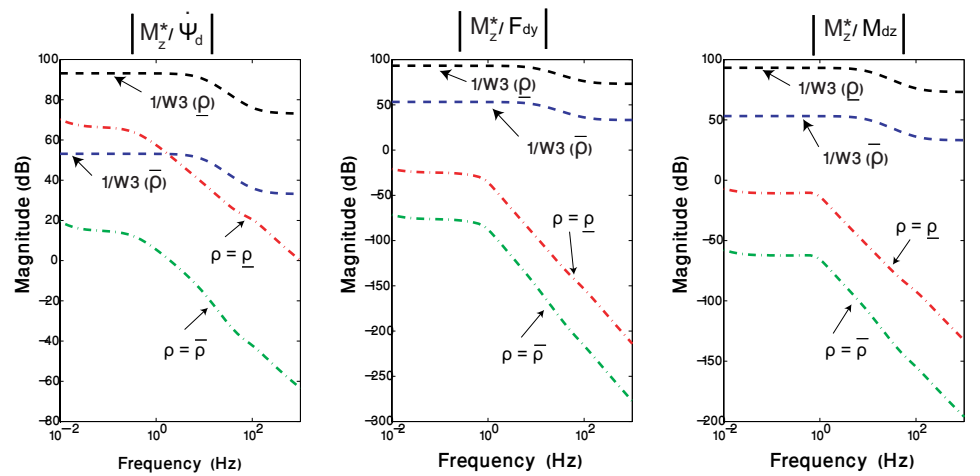

(c) Closed loop transfer functions between $M^{*}$ and exogenous inputs.
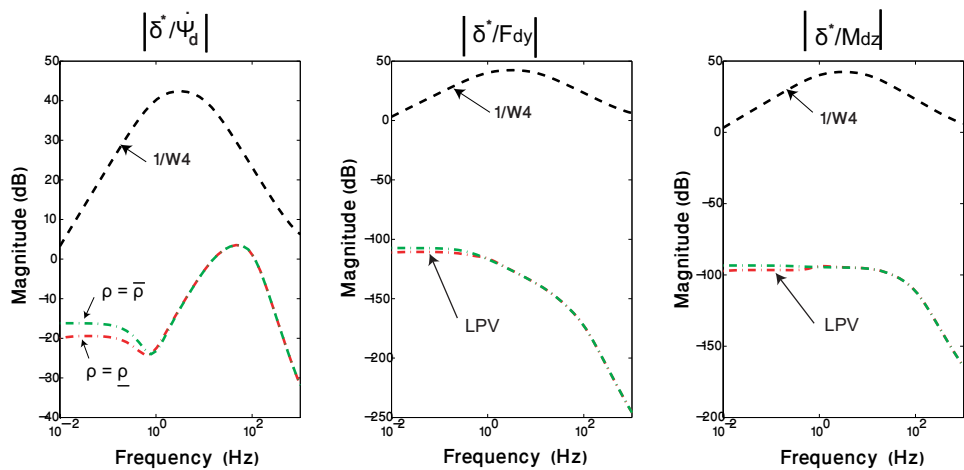

(d) Closed loop transfer functions between $\delta^{*}$ and exogenous inputs.

Figure 6: Closed loop transfer functions: $L P V\left(\right.$ red dashed $\left(\rho=10^{-5}\right)$, or green dashed $\left(\bar{\rho}=10^{-3}\right)$ ) synthesis results; Inverse of weighting functions (black dashed) of $1 / W_{1}, 1 / W_{2}, 1 / W_{3}$, and $1 / W_{4}$. 

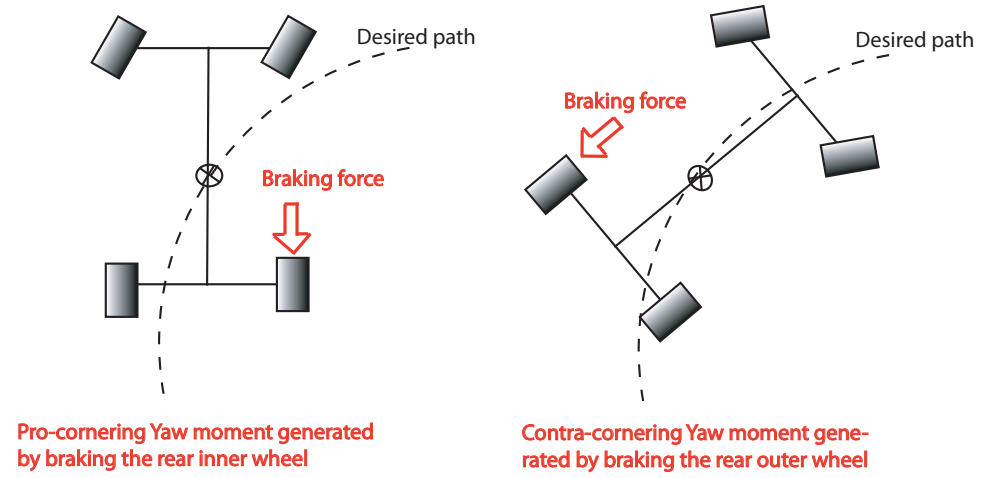

Figure 7: Schematics of selectively braking individual wheels in a right hand turn.

generate the control moment [1]. Another advantage of the scheme to apply the brake torque only at one wheel at a time, is that the vehicle is not as much decelerated as when brake torque is applied at more than one wheel to generate the same amount of yaw moment. In this study, to avoid overlapping with front steering actuators, only rear wheels are involved in the control law.

Based on the above analysis and assuming counterclockwise positive, the lower-level controller law is described as follows :

$$
\begin{cases}\dot{\psi}>0, \xi>0 \rightarrow \text { Brake rear left wheel: } & T_{b r l}^{*}=\frac{2 R M_{z}^{*}}{t_{r}} \\ \dot{\psi}<0, \xi>0 \rightarrow \text { Brake rear right wheel: } & T_{b r r}^{*}=\frac{-2 R M_{z}^{*}}{t_{r}} \\ \dot{\psi}>0, \xi<0 \rightarrow \text { Brake rear right wheel: } & T_{b r r}^{*}=\frac{-2 R M_{z}^{*}}{t_{r}} \\ \dot{\psi}>0, \xi<0 \rightarrow \text { Brake rear left wheel: } & T_{b r l}^{*}=\frac{2 R M_{z}^{*}}{t_{r}}\end{cases}
$$

where $\xi=\left|\dot{\psi}_{d}\right|-|\dot{\psi}|$. The schematics of selectively braking individual wheels is shown in figure 7 for the case of a right hand turn.

\subsection{Monitor: coordination $L P V$ strategy of steering and braking}

As the brake-based DYC technique is not desirable in normal driving situations because of its direct influence on the longitudinal dynamics, the aim of the monitor is to minimize the use of the braking. Consequently, the braking actuators must only be used when the vehicle goes toward instability. Since vehicle stability is directly related to the sideslip motion of the vehicle, judging the vehicle stability region is derived from the phase-plane $(\beta-\dot{\beta})$ method. A stability bound defined in [23] is used here, and is formulated as:

$$
\chi<1
$$

where $\chi=|2.49 \dot{\beta}+9.55 \beta|$ is the "Stability Index". Therefore, when the vehicle states move beyond the control boundaries and enter the unstable region, braking actuators will be involved to generate an 


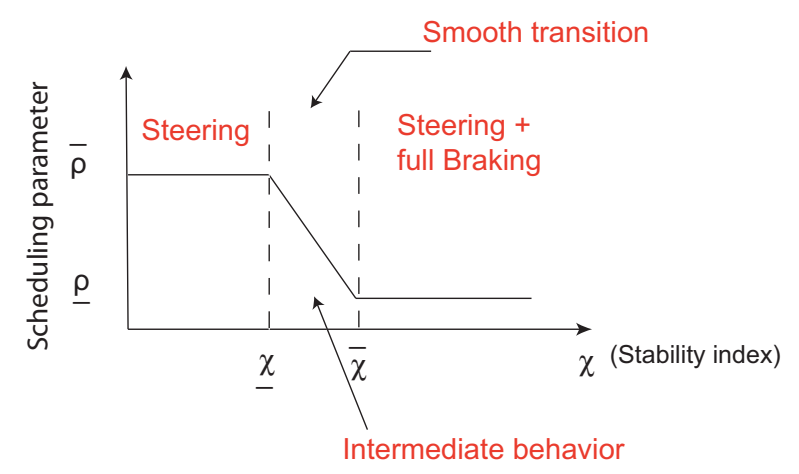

Figure 8: Control task selection according to the stability index variation.

additive corrective yaw moment, pulling the vehicle back into the stable region. According to [23], one of the significant benefits of this stability index, is that the reference region defined in (20) is largely independent of the road surface conditions and hence, the accurate estimation of the road surface coefficient of friction is not required.

Remember that the control task is also supposed to provide a seamless application of the direct yaw moment control when it is required. Hence, the scheduling parameter $\rho(\chi)$ can be defined as:

$$
\rho:= \begin{cases}\bar{\rho} & \text { if } \chi \leq \underline{\chi} \text { (steering control-steerability control task) } \\ \frac{\bar{\chi}-\chi}{\bar{\chi}-\underline{\chi}} \bar{\rho}+\frac{\chi-\underline{\chi}}{\bar{\chi}-\underline{\chi} \underline{\rho}}- & \text { if } \underline{\chi}<\chi<\bar{\chi} \text { (steering+braking) } \\ \underline{\rho} & \text { if } \chi \geq \bar{\chi} \text { (steering+full braking-stability control task) }\end{cases}
$$

where $\underline{\chi}=0.8$ (user defined) and $\bar{\chi}=1$. The control task selection is illustrated in figure 8 .

\subsection{Sideslip dynamics estimation}

To calculate the actual stability index $\chi$ defined in the previous subsection, the block called "Sideslip dynamics observer" (see figure 1) should evaluate $\dot{\beta}$ and $\beta$ in real-time:

- $\dot{\beta}$ can be reconstructed using available sensors, according to the following relationship:

$$
\dot{\beta}=\frac{a_{y}}{v_{x}}-\dot{\psi}
$$

where $a_{y}$ is the lateral acceleration and $v_{x}$ is the forward vehicle speed that can be approximated by the mean of the rear wheel velocities. 
- $\beta$ is not available using standard sensors, and thus, it must be estimated. The " $\beta$-estimation" is widely discussed in the literature, and many papers are concerned with that topic (see $[31,27,19,29])$. Here, the observer developed in $[12,14]$ has been used, which is suitable for real-time implementation.

\subsection{Actuator models}

The corrective steer angle and rear braking torques control signals can be generated via actuation systems. In this particular research, let us consider the following actuators:

- A steer-by-wire Active Steering (AS) system providing an additional steering angle, modeled as:

$$
\dot{\delta}^{+}=2 \pi f_{4}\left(\delta^{*}-\delta^{+}\right)
$$

where $f_{4}=10 \mathrm{~Hz}$ is the actuator cut-off frequency, $\delta^{*}$ and $\delta^{+}$are the steering controller and actuator outputs respectively. This actuator is bounded between $\left[-5^{\circ},+5^{\circ}\right]$.

- Brake-by-wire Electro Mechanical Braking (EMB) actuators providing a continuously variable braking torque. The EMB model is given by:

$$
\dot{T}_{b_{r j}}^{+}=2 \pi f_{2}\left(T_{b_{r j}}^{*}-T_{b_{r j}}^{+}\right)
$$

where $f_{2}=10 \mathrm{~Hz}$ is the actuator cut-off frequency, $T_{b_{r j}}^{*}$ and $T_{b_{i j}}^{+}$are the local braking controller and actuator outputs respectively. Note that in this paper, only the rear braking system is used to avoid coupling phenomena occurring with the steering system. This actuator control is limited between $[0,1200] \mathrm{Nm}$.

\section{Simulation results and analysis}

\subsection{Simulation framework}

The block labeled "Vehicle simulation model" represents a full nonlinear vehicle model. This model has been validated on a real experimental French car "Renault Mégane Coupé" within the frame of a collaborative work with the MIAM research team, Haute-Alsace University, France. The involved model will be used in simulation for validation purpose only. Note that the main interest in using the full vehicle model is that it allows to take into account load transfers, nonlinear tires behavior, suspension 
dynamics, slipping and sideslip angles that are essential factors which play a major role on the global chassis dynamics, especially in critical driving situations. For more information about the vehicle model, refer to [34].

To clarify the effects of the proposed integrated control scheme, both the vehicle dynamics with and without controllers are checked and compared through nonlinear time and frequency domain simulations. Various tests are carried out on the nonlinear complex vehicle model platform. In the following, the uncontrolled (or passive) vehicle responses are plotted in "dash", the controlled vehicle in "solid", and the yaw rate references in "dash-dot" lines.

\subsection{Time domain simulations}

Simulations of critical driving situations have been performed on the full non linear vehicle model to illustrate the benefits of the control scheme.

a) Double-lane-change maneuver on a dry road at $105 \mathrm{~km} / \mathrm{h}$ :

In the first simulation, the vehicle negociates a double-lane-change maneuver on a dry road $(\mu=$ 0.9). This maneuver is often used in the vehicle handling performance test. The vehicle speed equals $105 \mathrm{~km} / \mathrm{h}$.

Figure 9 shows the yaw rate response versus the steering input commanded by the driver. It can be deduced that the uncontrolled vehicle becomes rather unstable as the amplitude of the steering input becomes larger. On the other hand, the controlled output of the yaw rate is nearly converging to the output of the desired linear model. These results are confirmed by figure 10, where the yaw rate of the uncontrolled vehicle significantly lags the desired yaw rate, while the controlled vehicle closely tracks the desired yaw response. Comparisons between the sideslip angles and the lateral accelerations of the uncontrolled and controlled vehicles are illustrated in figures 11 and 12. The vehicle with integrated control achieves lower peaks for the lateral acceleration and sideslip angle in response to the steer input, compared to the uncontrolled vehicle. Consequently, the handling performances are much improved by the proposed controller.

The figure 13- 13(a) illustrates the $L P V$ integrated control action on the vehicle behavior. Figure 13(a) illustrates how the stability index, the dependency parameter $\rho$ and the generated corrective yaw moment $M_{z}^{*}$ evolve according to the driving situations. As stated before, when the stability index, $\chi$, is below 0.8 , only steering control is involved to enhance the handling performances. Indeed $\rho$ is equal to $\bar{\rho}$ and the corrective yaw moment is penalized and nearly zero. Conversely, when $\chi$ exceeds 0.8 , the 


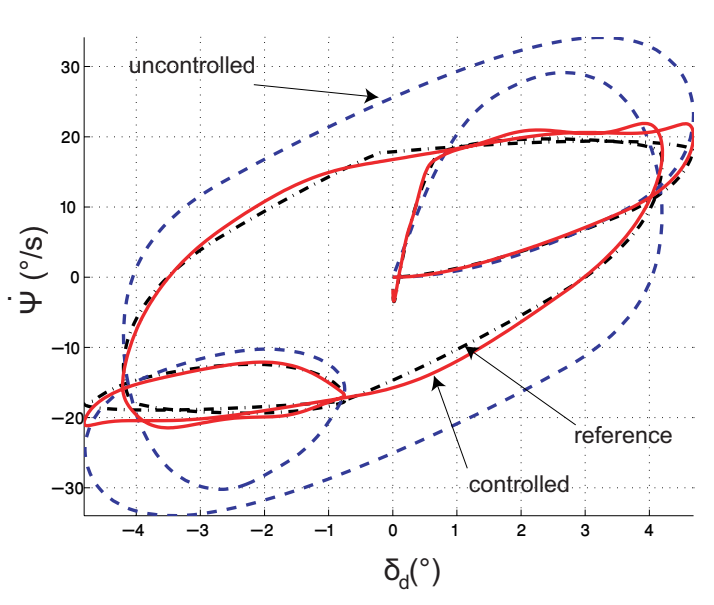

Figure 9: Responses of the yaw rates versus steering wheel angle for the double lane-change maneuver.

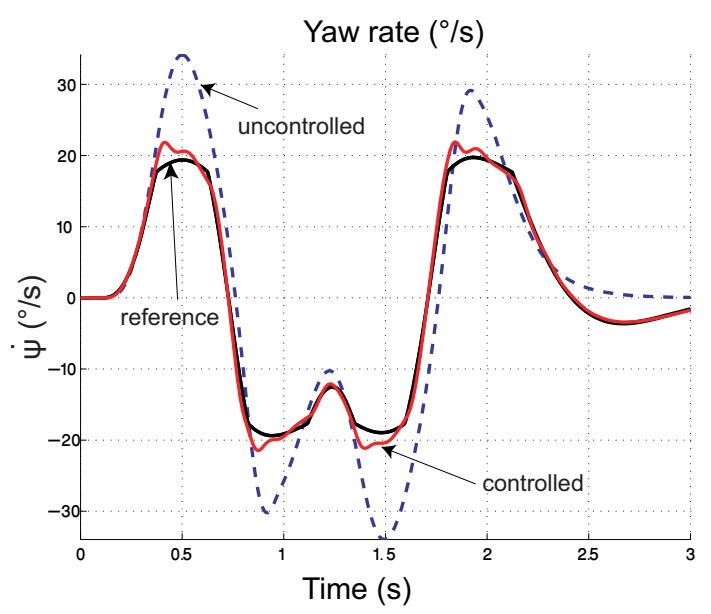

Figure 10: Yaw rates of the controlled and uncontrolled vehicles for the double lane-change maneuver.

braking system acts in addition to the active steering in order to keep the vehicle stable.

Figure 13(b) shows the generated corrective steering angle and the brake torques to enhance the lateral vehicle control. It is worth noting, that despite this test agressivity, actuators are far from saturation that may lead to instability. Also as said previously, the simple lower-level control strategy that activates the right or left braking torques could be replaced by more advanced ones since the second controller output is the yaw moment $M_{z}^{*}$. The trajectories of the controlled and passive vehicles given in figure 14 are compared to the ideal trajectory. It can be noticed that the controlled vehicle tracks better the desired path. The sideslip dynamic variation is reported in the phase-plane $(\beta-\dot{\beta})$ illustrated in figure 15. It is clear that the vehicle with the integrated control operates in the safety envelope defined in equation (20), during the whole test, while the passive vehicle enters the critical unsafe zone. Then controlled car is then brought back to a normal driving situation.

b) Steering maneuver at $80 \mathrm{~km} / \mathrm{h}$ on a wet road:

In the second simulation, the vehicle performs a steering maneuver at $80 \mathrm{~km} / \mathrm{h}$ on a slippery wet road $(\mu=0.6)$. Similar features found in the previous test can also be observed in this maneuver.

Figures 16 to 21 depict the dynamic responses of the uncontrolled and the controlled vehicle. Again, the proposed controller shows satisfactory tracking performances for the yaw rate and the desired trajectory, besides attenuation of the body sideslip angle. Therefore, the vehicle handling and stability are improved by the proposed integrated control system w.r.t corresponding passive vehicle, regardless of the road condition. Figure 20(a) gives the evolution of $\rho$ as function of $\chi$, and the generation of $M_{z}^{*}$ according to $\rho$ for this maneuver. As discussed in the previous test, when the stability index is below 0.8 , only steering actuator is involved to enhance the handling performance (this is the major case for 


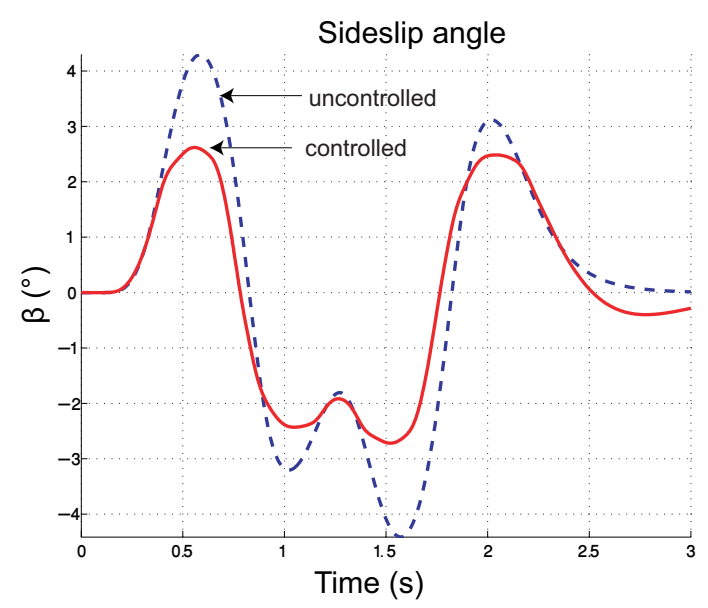

Figure 11: Sideslip angles of the controlled and uncontrolled vehicles for the double lanechange maneuver.

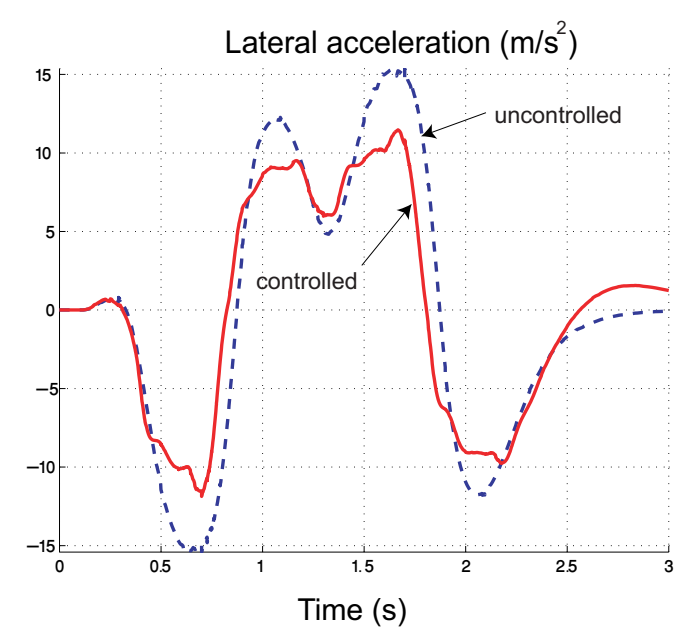

Figure 12: Lateral accelerations of the controlled and uncontrolled vehicles for the double lane-change maneuver.

this test), and when the stability index exceeds 0.8 , the braking system begins to work with the active steering system to keep the vehicle stable. The observations about enhancement of the vehicle lateral dynamics are also clarified in the $(\beta-\dot{\beta})$ diagram given in figure 22 . It shows that the braking system only acts when a dangerous situation is met, which avoids decreasing too much the vehicle speed.

c) Analysis of the roll behavior:

It is crucial to note that the "yaw control" indirectly influences the roll motion of the vehicle by reducing the lateral acceleration. Figures 23 and 24 show the Lateral Transfer Ratio (LTR) for both controlled and uncontrolled vehicles. The $L T R$, defined as a convenient method for supervising the vehicle's dynamic roll behavior [18], is the ratio of the difference between the sum of the left and right wheel loads to the sum of all wheel loads:

$$
L T R=\frac{F z_{l}-F z_{r}}{F z_{l}+F z_{r}}=\frac{\Delta F z_{l}}{F z_{l}+F z_{r}}
$$

where $F z_{l}$ and $F z_{r}$ are respectively the vertical loads on the left and right tires. The value of $L T R$ varies from -1 at the lift-off of the left wheel, tends toward 0 at no load transfer, and to 1 at the lift-off of the right wheel. From figures 23 and 24, it is clear that the controlled vehicles do have a smaller LTR coefficient. For the double-lane-change, it is deduced that the controlled vehicle is far from the rollover situation, while the uncontrolled vehicle tackles a dangerous situation with an LTR up to 0.85 .

\subsection{Frequency-domain simulations}

A "nonlinear" frequency analysis is done on the full nonlinear vehicle model by applying: 

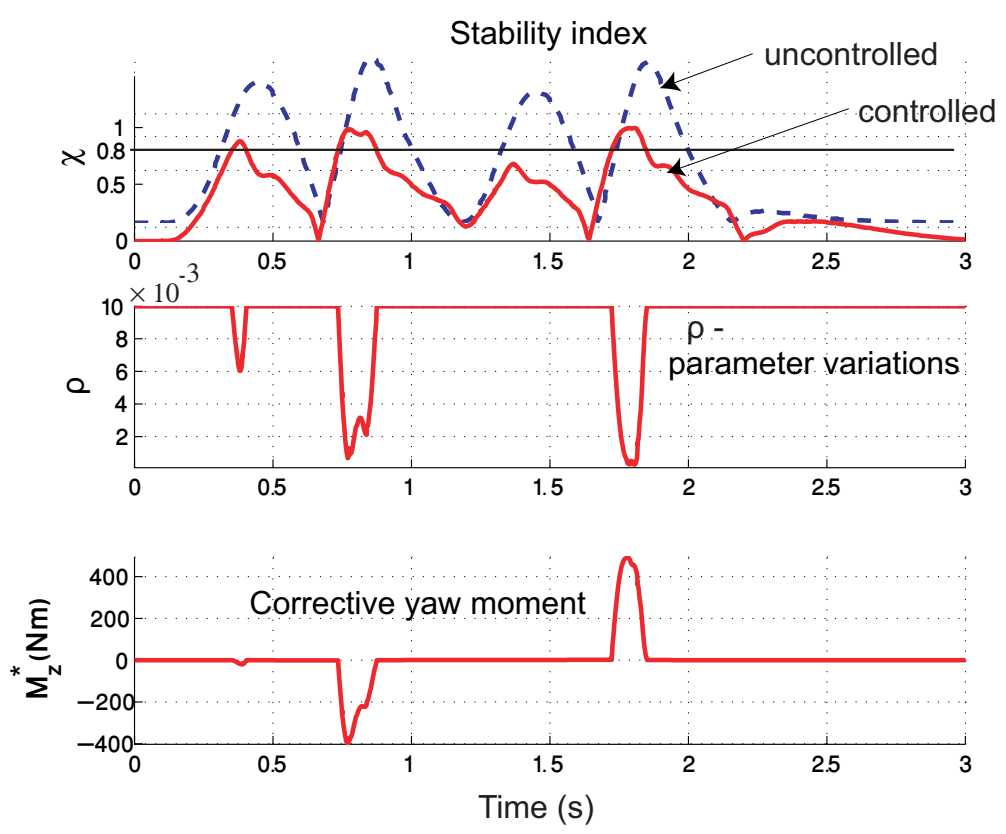

(a) $M_{z}^{*}$ and $\rho$ variations according to $\chi$ for the double lane-change maneuver.
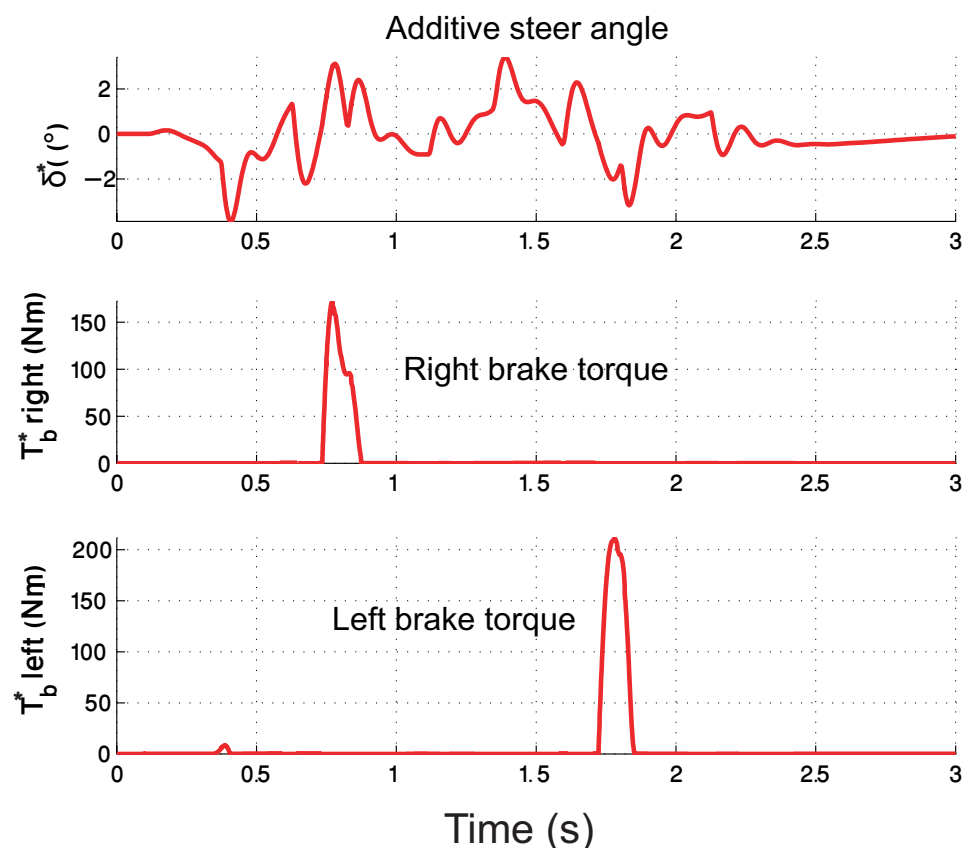

(b) Control signals generated by the controller for the double lane-change maneuver.

Figure 13: $L P V$ integrated control action for the double lane change test. 


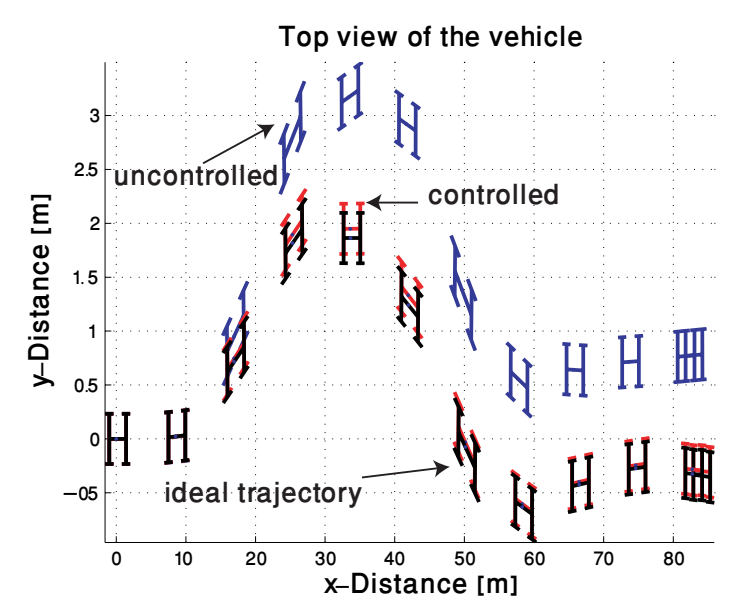

Figure 14: Trajectories of the controlled and uncontrolled vehicles for the double lane-change maneuver.

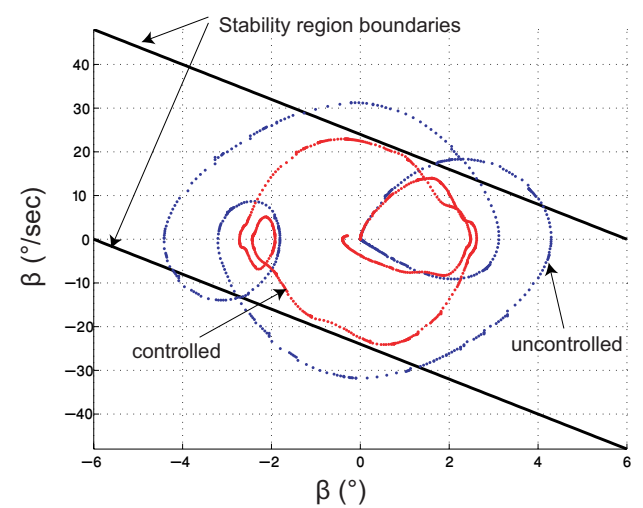

Figure 15: Evolutions of the controlled and uncontrolled vehicles in the phase-plane $\beta-\dot{\beta}$ for the double lane-change maneuver.

1. Test A: a sinusoidal disturbance, $M_{d z}$ over varying frequencies : $M_{d z}(t)=A \sin (2 \pi f t)$, where $A=2000 N . m$ and $f \in[0.1 ; 3] \mathrm{Hz}$. In this test, the driver is assumed to drive the vehicle in a straight line with a speed about $90 \mathrm{~km} / \mathrm{h}$ on a dry and a wet road, respectively. The objective of this simulation is to verify the capability of the controller to reject disturbances.

2. Test B: a sinusoidal steering angle, $\delta_{d}(t)=B \sin (2 \pi f t)$, where $B=4^{\circ}$ and $f \in[0.1 ; 1] H z$. This test corresponds to slalom maneuvers with different frequencies. The test is carried out on a dry and a wet road, with a speed about $50 \mathrm{~km} / \mathrm{h}$. This simulation goal is to check the controller response with respect to fast steering actions.

The frequency responses of the controlled and passive vehicles corresponding to Test $\mathbf{A}$ and $\mathbf{B}$ are illustrated in figures 25 and 26, respectively. The figures clearly show that the proposed control design enhances the main critical car dynamics by decreasing the $\left|\beta / M_{d z}\right|$ and $\left|e_{\psi} / M_{d z}\right|$ spectrum. Thus, the integrated control proves its advantages with respect to a passive vehicle, whatever the road state (dry or wet). These results are in good accordance with time-domain simulation tests.

\section{Conclusion}

The major contribution of this paper is concerned by the development and the analysis of an integrated control strategy involving AFS (Active Front Steering, using the front steering command) and DYC (Direct Yaw Control, using differential rear braking), based on a gain-scheduled controller able to im- 


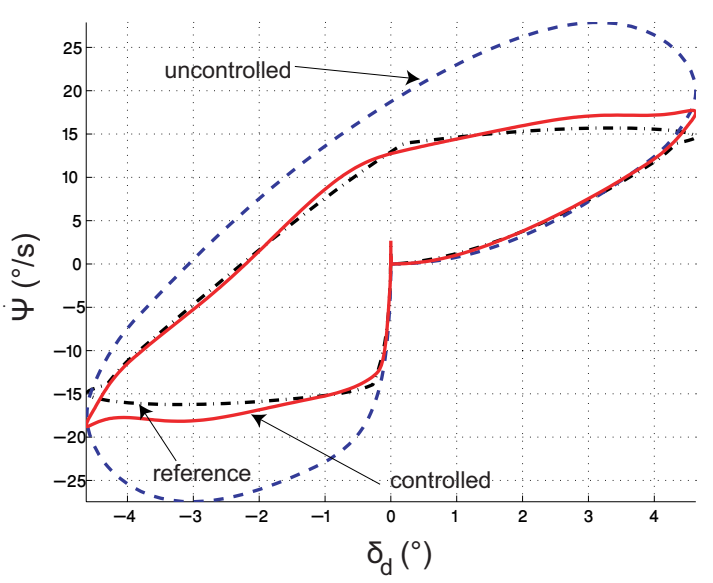

Figure 16: Responses of the yaw rates versus steering wheel angle for the steering maneuver.

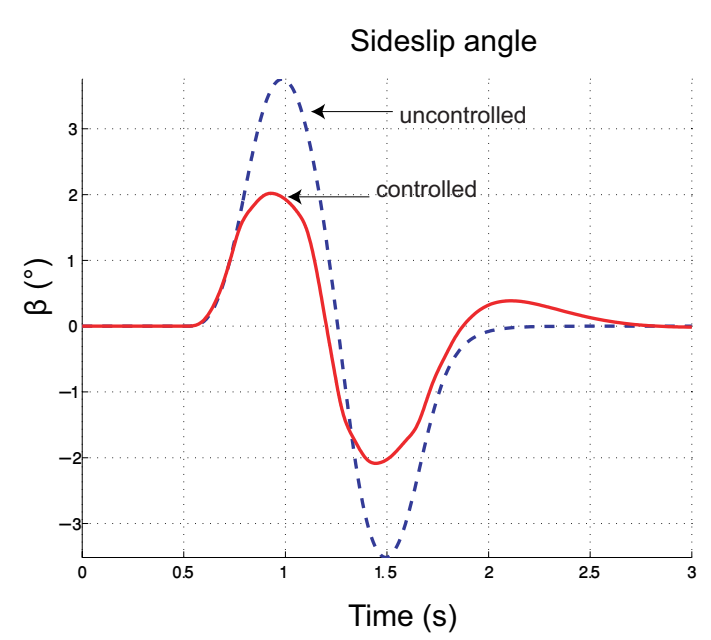

Figure 18: Sideslip angles of the controlled and uncontrolled vehicles for the steering maneuver.

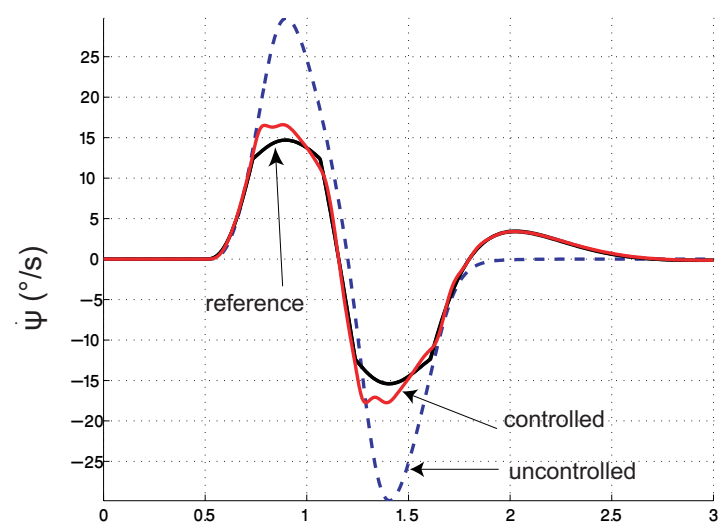

Figure 17: Yaw rates of the controlled and uncontrolled vehicles for the steering maneuver.

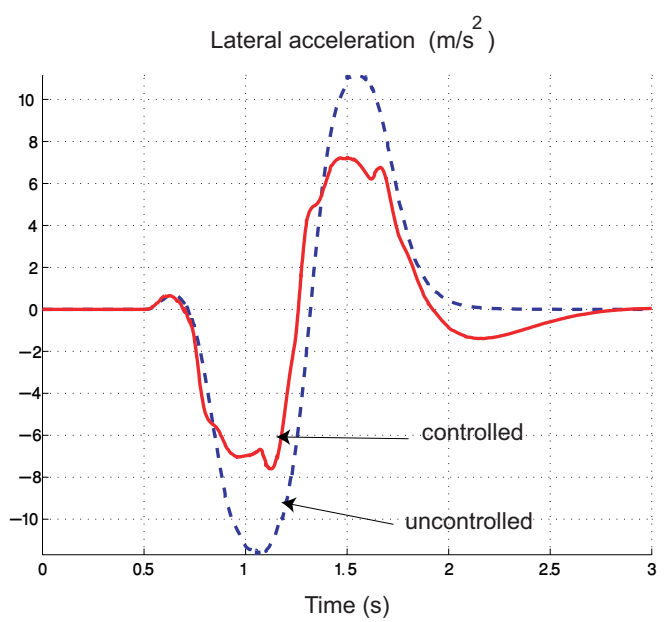

Figure 19: Lateral accelerations of the controlled and uncontrolled vehicles for the steering maneuver. 

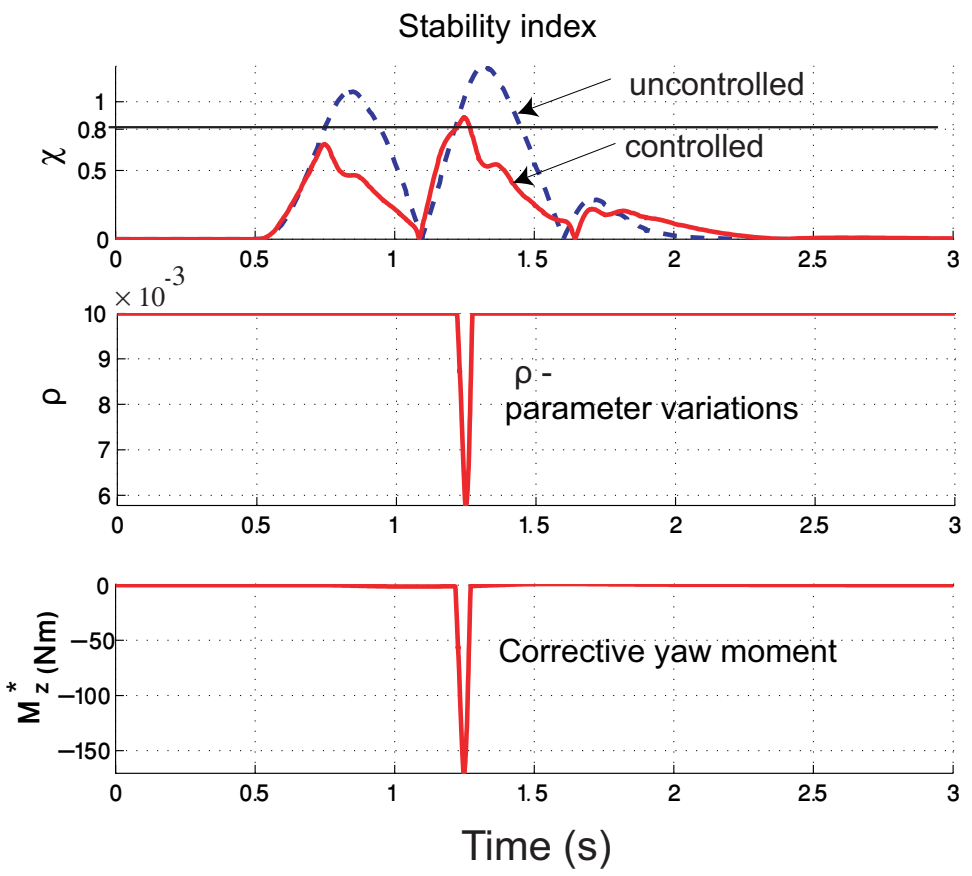

(a) $M_{z}^{*}$ and $\rho$ variations according to $\chi$ for the steering maneuver.
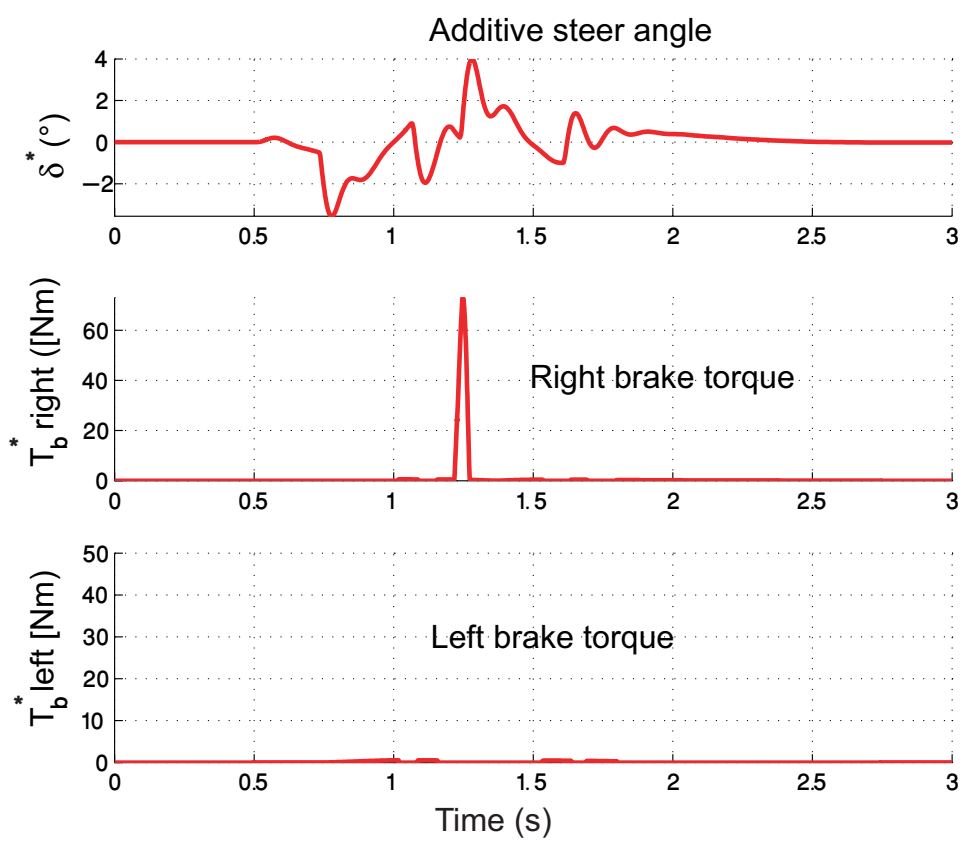

(b) Control signals generated by the controller for the steering maneuver.

Figure 20: $L P V$ integrated control action for the steering maneuver. 


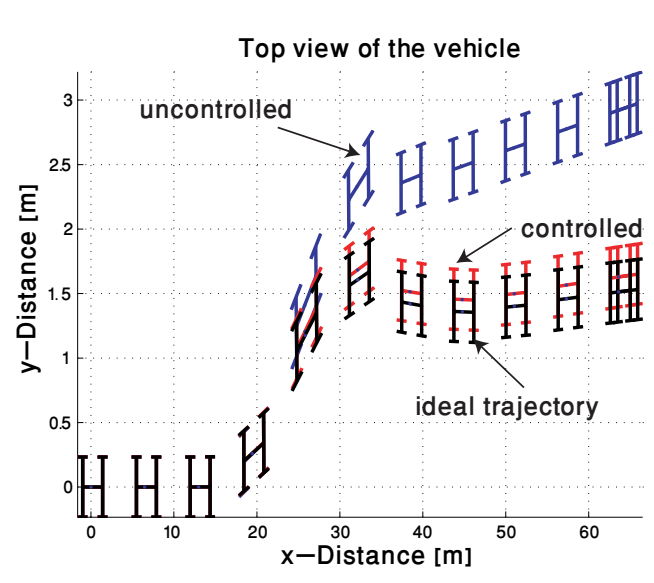

Figure 21: Trajectories of the controlled and uncontrolled vehicles for the steering maneuver.

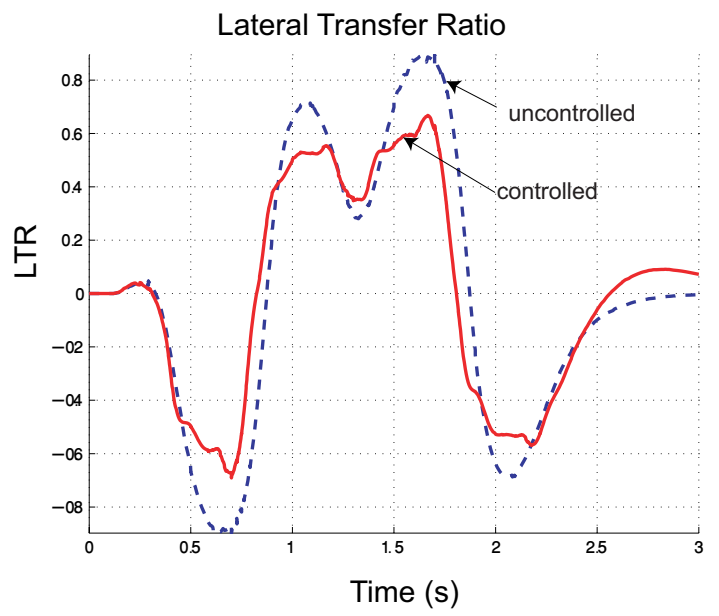

Figure 23: $L T R$ variations for the double lanechange maneuver.

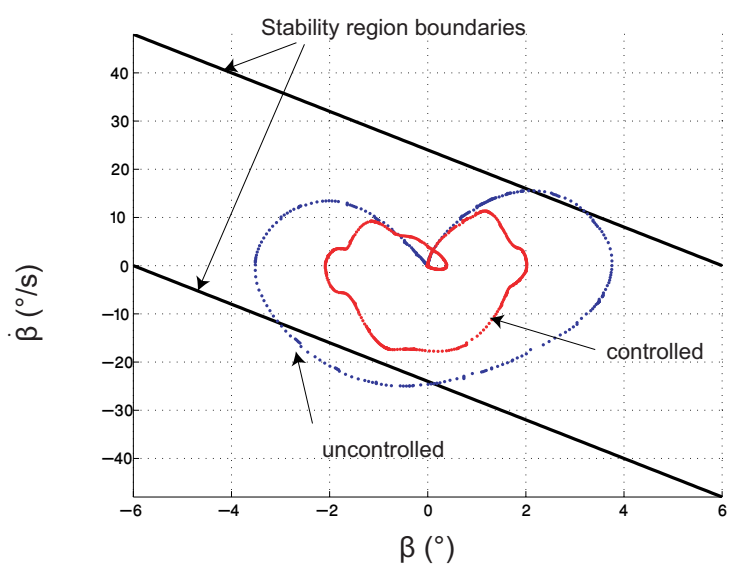

Figure 22: Evolutions of the controlled and uncontrolled vehicles in the phase plane $\beta-\dot{\beta}$ for the steering maneuver.

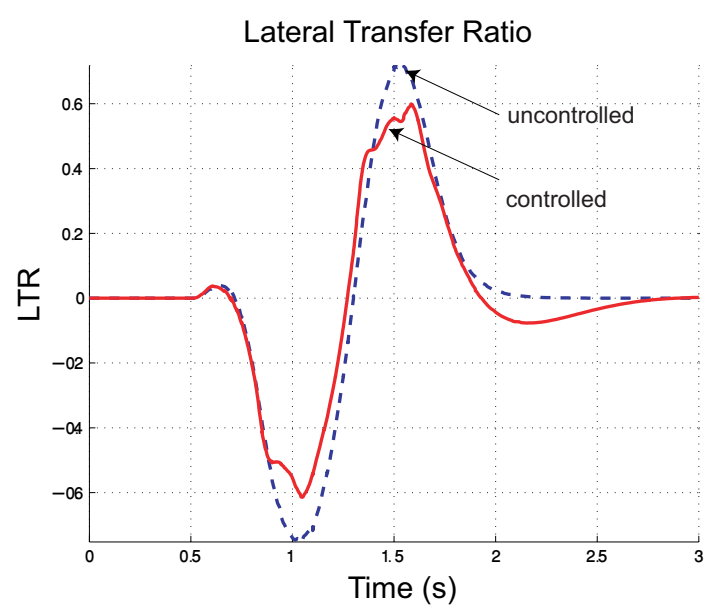

Figure 24: $L T R$ variations for the steering maneuver. 
prove stability, handling and comfort in a four-wheeled vehicle. Thus, a hierarchical control structure is formalized:

- the upper-level LPV controller manages the $A F S$ and $D Y C$ integration;

- the low-level controller performs the desired direct yaw moment by exploiting the quasi-static rotational dynamics of the wheel, and decides which brake should be used to carry-out the desired yaw moment and selects the proper intensity of the corresponding braking force.

A model reference approach is adopted in the overall system since the desired yaw rate which feds the upper-level controller is computed online using a linear single-track model of the vehicle lateral dynamic, in order to restrain the behavior of the vehicle inside its linear region. The bicycle model is also used for the synthesis of the high-level controller. The requirements for the high-level controller are specified in terms of $\mathscr{H}_{\infty}$ performances. This allows a simple description of the generalized plant and eases the synthesis of the controller. Moreover, by introducing a varying parameter in the weighting functions which characterize the performance objectives, the controller handles the trade-off between the AFS and DYC control, while guaranteeing the stability of the overall system.

The gain-scheduled controller is designed following the $L P V$ approach. In order to manage the trade-off between $A F S$ and $D Y C$, the scheduling parameter depends on the stability index function of the sideslip dynamics. This way, the steering action can be adopted in stable conditions, while the $A F S$ and $D Y C$ acts together in unsafe dynamic situations, where the $A F S$ is not able to provide good stability performance. A smooth transition between both controllers is guaranteed by the shape of the function.

Finally, time and frequency domain simulations assess the performances of the overall control system in various maneuver conditions.

The general structure of the proposed control scheme does not involve any online optimization process, thus it may be implemented on a real car, and tested for robustness analysis with respect to real driving situations. This work could be lead to many extensions or related works such as:

- consider additional degrees-of-freedom as active rear steering and front braking

- improve the braking torque distribution through different strategies such as in $[6,8]$

- integrate semi-active suspensions in the control scheme to avoid dangerous rollover situations as preliminary studied in [34].

- consider a more complete control-oriented $L P V$ vehicle model depending also on the vehicle forward speed, vehicle total mass... 

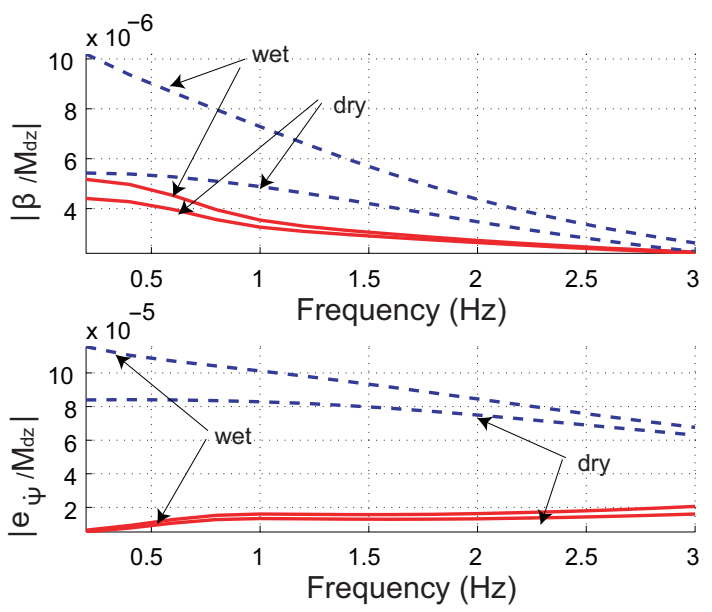

Figure 25: Test A: frequency responses for $\left|\beta / M_{d z}\right|$ and $\left|e_{\psi} / M_{d z}\right|$; controlled vehicle (solid line) and uncontrolled vehicle (dash line).
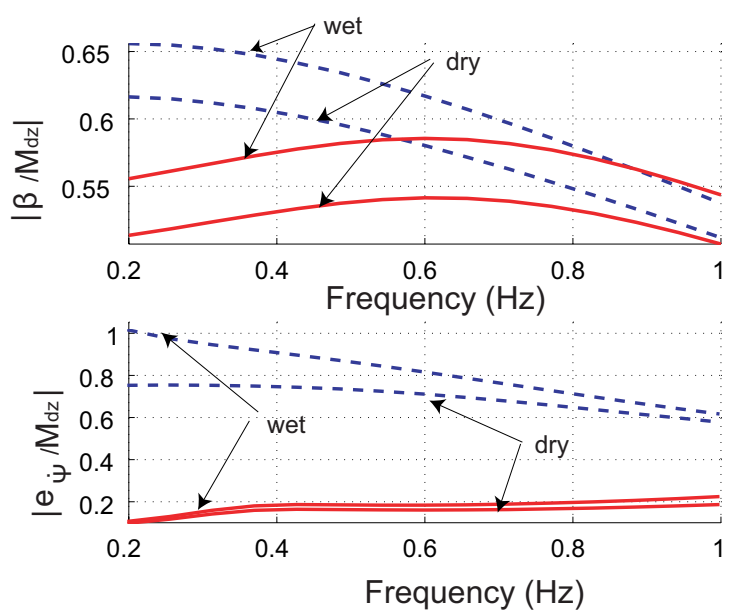

Figure 26: Test B: frequency responses for $\left|\beta / M_{d z}\right|$ and $\left|e_{\psi} / M_{d z}\right|$; controlled vehicle (solid line) and uncontrolled vehicle (dash line).

\section{Acknowledgments}

This work was supported by the French national project INOVE/ ANR 2010 BLAN 0308.

\section{References}

[1] Anwar S. Yaw stability control of an automotive vehicle via generalized predictive algorithm. Proceedings of the American Control Conference, Portland, USA, 2005.

[2] Apkarian P, Gahinet P. A convex characterization of gain scheduled $H_{\infty}$ controllers. IEEE Transaction on Automatic Control 1995; 40: 853-864.

[3] Apkarian P, Gahinet P, Beker G. Self-scheduled $\mathscr{H}_{\infty}$ control of linear parameter-varying systems: a design example. Automatica 1995; 31: 1251-1262.

[4] Boada BL, Boada MJL, Diaz V. Fuzzy-logic applied to yaw moment control for vehicle stability. Vehicle System Dynamics 2005; 43(10): 753-770.

[5] Burgio G., Girelli C., Alessandri D., Palandri J.. Active Rear Steering System And Controller Design To Improve Vehicle Driving And Handling Behavior, FISITA 2010, World Automotive Congress, 30 May - 4 June, Budapest, Hungary. 
[6] Canale M, Fagiano L, Milanese M, Borodani P. Robust vehicle yaw control using an active differential and IMC techniques. Control Engineering Practice 2007; 15(8): 923-941.

[7] Canale M, Fagiano L, Ferrora A, Vecchio C. Vehicle yaw control via second order sliding mode technique. IEEE Industrial Electronics 2008; 55(11): 3908-3916.

[8] Corno M., Tanelli M., Boniolo I., and Savaresi S.M., "Advanced yaw control of fourwheeled vehicles via rear active differential braking", Proceedings of the 48th IEEE Conference on Decision and Control, 2009, pp. 5176 - 5181.

[9] Corno M., Active Stability Control Systems Design for Road Vehicles, PhD thesis, POLITECNICO DI MILANO, Dipartimento di Elettronica e Informazione, 2008

[10] Di Cairano S., Tseng H.E., Bernardini D., Bemporad A. "Steering Vehicle Control by Switched Model Predictive Control", IFAC Symposium Advances in Automotive Control 2010, Munich, Germany

[11] Di Cairano S., Tseng, H.E.. "Driver-assist steering by active front steering and differential braking: design, implementation and experimental evaluation of a switched model predictive control approach". Proceedings of the 49th IEEE Conference on Decision and Control, pp. 2886-2891, Atlanta, USA, 2010.

[12] Doumiati M, Victorino A, Lechner D, Baffet G, Charara A. Observers for vehicle tyre/road forces estimations: experimental validation. Vehicle System Dynamics 2010; 48(11): 13451378 .

[13] Doumiati M., Sename O., Martinez J., Dugard L. and Poussot-Vassal C., "Gain-scheduled LPV/Hinf controller based on direct yaw moment and active steering for vehicle handling improvements", Proceedings of the 49h IEEE Conference on Decision and Control, Atlanta, USA, 2010.

[14] Doumiati M, Victorino A, Charara A, Lechner D. Onboard real-time estimation of vehicle lateral tire-forces and sideslip angle. IEEE Transactions on Mechatronics 2011; 16(4): 601-614.

[15] Dugoff H, Francher PS, Segel L. An analysis of tire traction properties and their influence on vehicle dynamic performance. SAE Transactions 1970; 79: 341-366. 
[16] Falcone P, Borrelli F, Asgari J, Tseng HE, Hrovot D. Predictive active steering control for autonomous vehicle systems. IEEE Transactions on Control Systems Technology 2007; 15(3): 566-580.

[17] Falcone P, Borrelli F, Tseng H.E, Asgari J, , Hrovot D. "Linear time-varying model predictive control and its application to active steering systems: Stability analysis and experimental validation". International Journal Of Robust And Nonlinear Control, vol 18, pp 862-875, 2008

[18] Gaspar P, Szabo Z, Bokor J. A fault-tolerant vehicle control design. Proceedings of the 17th IFAC World Congress, Seoul, Korea, June 2008.

[19] Grip HF, Imsland L, Johansen TA, Fossen TI. Nonlinear vehicle sideslip estimation with friction adaptation. Automatica 2008; 44(3): 611-622.

[20] Guvenç BA, Acarman T, Guvenç L. Coordination of steering and individual wheel braking actuated vehicle yaw stability control. Proceedings of the IEEE Intelligent Vehicle Symposium, pp. 288-293, 2003.

[21] Guvenç BA, Bunte T, Odenthal D, Guven L. Robust two degree-of-freedom vehicle steering controller design. IEEE Transactions on Control System Technology 2004; 12(4): 627-636.

[22] Guvenc, B.A.; Guvenc, L.; Karaman, S.; , "Robust Yaw Stability Controller Design and Hardware-in-the-Loop Testing for a Road Vehicle," Vehicular Technology, IEEE Transactions on , vol.58, no.2, pp.555-571, Feb. 2009

[23] He J, Crolla DA, Levesley MC, Manning WJ. Coordination of active steering, driveline, and braking for integrated vehicle dynamics control. Proc. Inst. Mech Engineers, PartD: Automobile Engineering 2006; 220 (10): 1401-1420.

[24] Hirano Y, Fukatani K. Development of robust active rear steering control. Proceedings of the 3rd Int. Symposium on Advanced Vehicle Control, AVEC'96, pp. 359-375, 1996.

[25] Horiuchi S, Yuhara N, Takei A. Two degree of freedom/ $\mathscr{H}_{\infty}$ controller synthesis for active four wheel steering vehicles. Vehicle System Dynamics Supplement 1996; 25: 275-292.

[26] Liang W., Yu H., McGee R., Kuang M., Medanic J.. Vehicle Pure Yaw Moment Control Using Differential Tire Slip 2009 American Control Conference, St. Louis, MO, USA, June 10-12, 2009 
[27] Lin-Hui Z, Zhi-Yuan L, Hong C. Design of a nonlinear observer for vehicle velocity estimation and experiments. IEEE Transactions on Control Systems Technology 2010; 18(3): 545-558.

[28] Lofberg J. YALMIP: a toolbox for modeling and optimization in MATLAB. Proceedings of the CACSD Conference, Taipei, Taiwan, 2004.

[29] Panzani G, Corno M, Tanelli M, Zappavigna A, Savaresi SM, Fortina A, Campo S. Designing On-Demand Four-Wheel-Drive Vehicles via Active Control of the Central Transfer Case," IEEE Transactions on Intelligent Transportation Systems, 2010; 11(4): 931-941.

[30] Park JH. $H_{\infty}$ direct yaw-moment control with brakes for robust performance and stability of vehicles. JSME International Journal, series C 2001; 44(2): 404-413.

[31] Piyabongkarn D, Rajamani R, Grogg JA, Lew JY. Development and experimental evaluation of a slip angle estimator for vehicle stability control. IEEE Transactions on Control Systems Technology 2009; 17(1): 78-88.

[32] Poussot-vassal C, Sename O, Dugard L. A $L P V / \mathscr{H}_{\infty}$ global chassis controller for handling improvements involving braking and steering systems. Proceedings of the 47th IEEE Conference on Decision and Control, CDC'08, Cancun, Mexico, December 2008.

[33] Poussot-Vassal, C.; Sename, O.; Dugard, L.; Savaresi, S., "Vehicle Dynamic Stability Improvements Through Gain-Scheduled Steering and Braking Control", Vehicle System Dynamics, vol 49, nb 10, pp 1597-1621, 2011.

[34] Poussot-vassal C, Sename O, Dugard L, Gaspar P, Szabo Z, Bokor J. Attitude and handling improvements through gain-scheduled suspensions and brakes control. Control Engineering Practice $2011 ; 19: 252-263$.

[35] Rajamani R. Vehicle dynamics and control. Springer New York 2006.

[36] Ray LR. Nonlinear state and tire force estimation for advanced vehicle control. IEEE Transactions on Control Systems Technology 1995; 3(1): 117-124.

[37] Rodic AD, Vukobratovic MK. Contribution to the integrated control synthesis of road vehicles. IEEE Transactions on Control Systems Technology 1999; 7(1): 64-78. 
[38] Scherer C, Gahinet P, Chilali M. Multiobjective output-feedback control via LMI optimization. IEEE Transactions on Automatic Control 1997; 40: 896-911.

[39] Schiebahn M, Zegalaar PWA, Lakehal-Ayat M, Hoffman O. The yaw torque influence of active systems and smart actuators for coordinated vehicle dynamics control. Vehicle System Dynamics 2010; 48(11): 1269-1284.

[40] Selby M, Manning WJ, Brown MD, Crolla DA. A comparison of the relative benefits of active front steering and active rear steering when coordinated with direct yaw moment control. Proceedings of ASME Int. Mechanical Engineering Congress and Exposition, pp. 1-6, 2001.

[41] Skogestad S, Postlethwaite I. Multivariable feedback control, analysis and design. Wiley 2007.

[42] Sturm JF. Using seDuMi 1.02, a Matlab toolbox for optimization over symmetric cones. Optimization Methods and Software 11-12, Special issue on Interior Point Methods 1999; 625-653.

[43] Tjonnas J, Johansen TA. Stabilization of Automotive Vehicles Using Active Steering and Adaptive Brake Control Allocation. IEEE Transactions on Control Systems Technology 2010; $18(3): 545-558$.

[44] Yang X, Wang Z, Peng W. Coordinated control of AFS and DYC for vehicle handling and stability based on optimal guaranteed cost theory. Vehicle System Dynamics, 2009; 47(1): 5779.

[45] Yoon J, Yim S, Cho W, Koo B, Yi K. Design of an unified chassis controller for rollover prevention, manœuvrability and lateral stability. Vehicle System Dynamics 2010; 48(11): 1247-1268. 